\title{
Stereodivergent Strategy for Total Syntheses of Antirhine Alkaloids
}

\author{
Eunjoon Park, ${ }^{\mathrm{a},}$ Cheolwoo Bae, ${ }^{\mathrm{a},}$ Cheon-Gyu Cho, ${ }^{\mathrm{b}}$ Cheol-Hong Cheon ${ }^{\mathrm{a}, *}$ \\ ${ }^{a}$ Department of Chemistry, Korea University, 145 Anam-ro, Seongbuk-gu, Seoul 02841, Republic of Korea \\ ${ }^{b}$ Department of Chemistry, Hanyang University, 222 Wangsimni-ro, Seongdong-gu, Seoul 04763, Republic of Korea
}

\section{Table of Contents}

1. Stereochemistry at the C-20 Position: Monitoring Thermal Elimination of Sulfoxide 25 ----------------------- S2

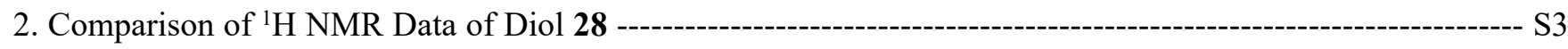

3. Facial Selectivity of Alkylation of Enolate of $\mathbf{2 3}$

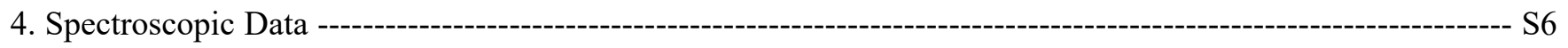




\section{Stereochemistry at the C-20 Position: Monitoring Thermal Elimination of Sulfoxide 25}

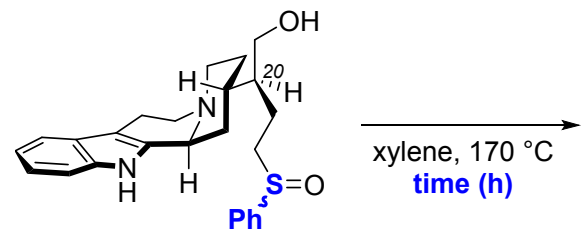

$25(\mathrm{dr}=3: 1)$

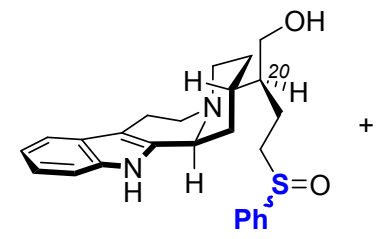

$25(\mathrm{dr}=3: 1)$

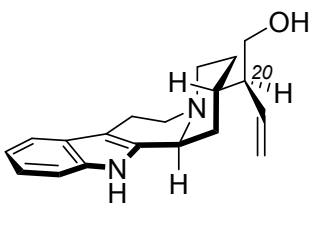

20-epi-antirhine (2)

To understand the thermal elimination of $\mathbf{2 5}$, leading to the exclusive formation of 20-epi-antirhine (2), we monitored the thermal elimination of the sulfoxide of alcohol 25. It was found that the ratio of the two diastereomers of alcohol $\mathbf{2 5}$ remained constant after $4 \mathrm{~h}$. Furthermore, all the alcohol 25 was completely converted into the desired 20-epi-antirhine (2) after $18 \mathrm{~h}$ and the yield of the isolated 20-epi-antirhine (2) after thermal elimination was higher than the amount of the major diastereomer (The diastereomeric ratio of the sulfoxide $\mathbf{2 5}$ was highlighted with blue color in the second line of the NMR below).

These results suggested that the possibility of the kinetic elimination from only one of the two diastereomers could be ruled out, and thus we concluded that the two diastereomers of Michael adduct $\mathbf{2 5}$ would result from the stereochemistry on the sulfur atom, not from carbon atom at the C-20 position. The reaction of enolate of $\mathbf{2 3}$ with vinyl sulfoxide would take place in highly stereoselective manner with excellent the $20-\alpha$-H selectivity.

\section{${ }^{1} \mathrm{H}$ NMR Spectra of a Reaction Mixture of Sulfoxide 25}

Starting sulfoxide $\mathbf{2 5}$ was highlighted with blue color and so was the product (2; 20-epi-antirhine) with red color.

(a) After $0 \mathrm{~h}$

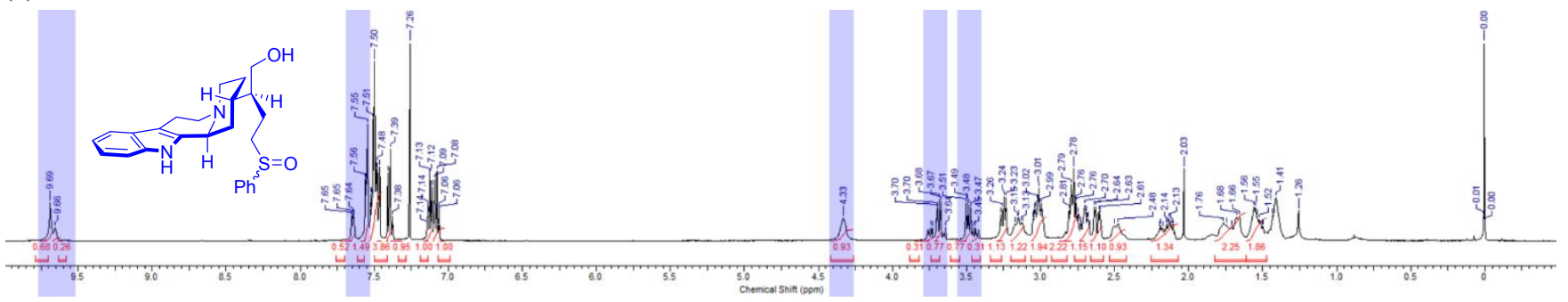

(b) After $4 \mathrm{~h}$

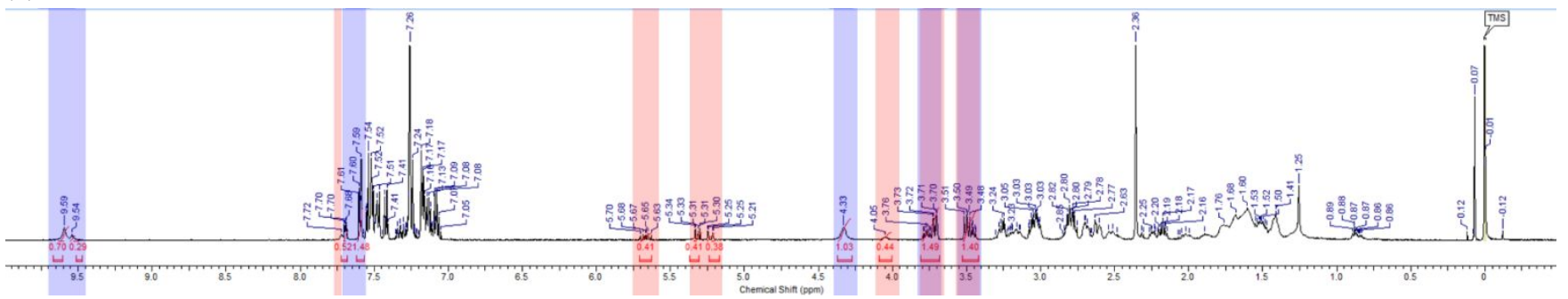

(c) After $18 \mathrm{~h}$

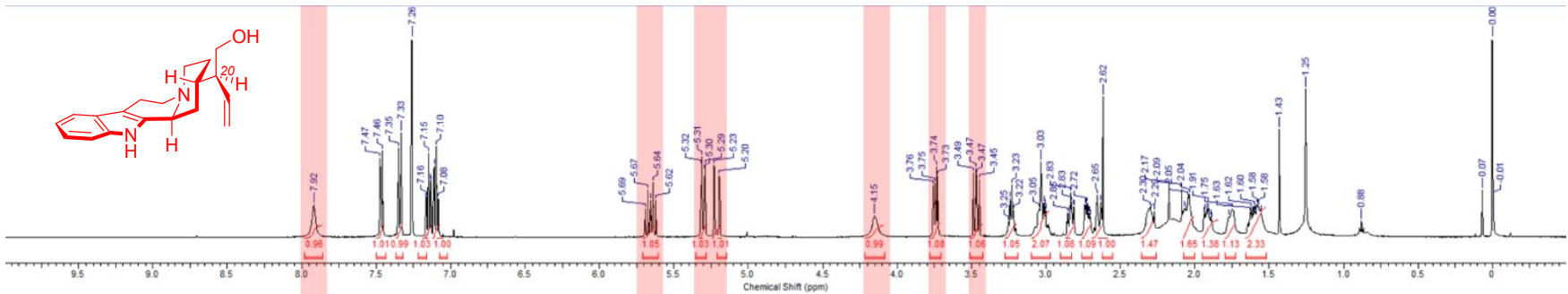




\section{Comparison of ${ }^{1} \mathrm{H}$ NMR Data of Diol 28}

Since diol 28 was employed as a key intermediate in the Takano's synthesis of antirhine (1), ${ }^{1}$ we compared ${ }^{1} \mathrm{H}$ NMR data of the resulting diol, $\mathbf{2 8}$ or 20-epi-28, with one reported in the literature to determine the relative stereochemistry of $\mathbf{2 8}$ (See below Table). However, since there was a large discrepancy between one in the literature and ones obtained from each isomer, we were not able to determine the stereochemistry at the C-20 position of the resulting diol 28.

\begin{tabular}{|c|c|c|c|}
\hline Attribution & $\begin{array}{c}\delta(\mathrm{ppm}) \\
\text { (Takano's group) }^{\mathbf{1}}\end{array}$ & $\begin{array}{c}\delta(\mathrm{ppm}) \\
\text { Compound } 28\end{array}$ & $\begin{array}{c}\delta(\mathrm{ppm}) \\
\text { Compound 20-epi-28 }\end{array}$ \\
\hline $\begin{array}{l}\mathrm{H}-18 \\
\mathrm{H}-21\end{array}$ & $3.98-3.40(\mathrm{~m}, 4 \mathrm{H})$ & $\begin{array}{l}3.78-3.58(\mathrm{~m}, 3 \mathrm{H}) \\
3.58-3.47(\mathrm{~m}, 1 \mathrm{H})\end{array}$ & $\begin{array}{c}3.79(\mathrm{dd}, 1 \mathrm{H}) \\
3.72-3.61(\mathrm{~m}, 3 \mathrm{H})\end{array}$ \\
\hline $\mathrm{H}-3$ & $4.15(\mathrm{br}, 3 \mathrm{H})$ & $4.28(\mathrm{br}, 1 \mathrm{H})$ & $4.40(\mathrm{br}, 1 \mathrm{H})$ \\
\hline $\begin{array}{c}\text { H-9, H-10, } \\
\text { H-11, H-12 } \\
\text { (Aromatic) }\end{array}$ & $7.60-6.90(\mathrm{~m}, 4 \mathrm{H})$ & $\begin{array}{c}7.40(\mathrm{~d}, 1 \mathrm{H}) \\
7.33(\mathrm{~d}, 1 \mathrm{H}) \\
7.13-7.01(\mathrm{~m}, 2 \mathrm{H})\end{array}$ & $\begin{array}{c}7.47(\mathrm{~d}, 1 \mathrm{H}) \\
7.36(\mathrm{~d}, 1 \mathrm{H}) \\
7.17-7.06(\mathrm{~m}, 1 \mathrm{H}) \\
\end{array}$ \\
\hline 1-H (Indole NH) & $9.02(\mathrm{br}, 1 \mathrm{H})$ & $9.31(\mathrm{br}, 1 \mathrm{H})$ & $8.64(\mathrm{br}, 1 \mathrm{H})$ \\
\hline
\end{tabular}

${ }^{1}$ S. Takano, M. Takahashi, K. Ogasawara, J. Am. Chem. Soc. 1980, 102, 4282-4283. 


\section{Facial Selectivity of Alkylation of Enolate of 23}

\section{3-1. Effect of Alkoxy Group in Carbamate in Compound 23 and Derivatives}
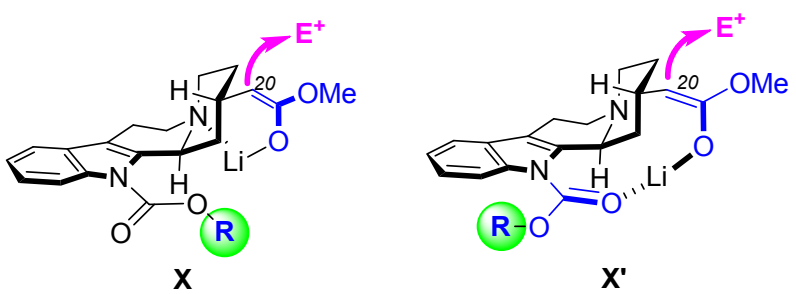

To determine a more plausible transition state for alkylation of enolate derived from $\mathbf{2 3}$ with an electrophile between transition states $\mathbf{X}$ and $\mathbf{X}$ ', we explored the facial selectivity of reactions of methoxymethyl (MOM) chloride with enolates derived from compound 23 and its derivatives carrying a different carbamate protecting group. According to our proposed transition state $\mathbf{X}$ (shown in Scheme 14), the size of an alkoxy group (highlighted in green in the below Scheme) in a carbamate would play some role in the facial selectivity; as the size of alkoxy group in the carbamate becomes larger, the facial selectivity would increase. On the other hand, if lithium cation were chelated with both the oxygen in the enolate and oxygen atom in the carbonyl of a carbamate in transition state $\mathbf{X}^{\prime}$ (as shown in ref 25), the facial selectivity might show little dependence on the size of carbamate since the alkoxy group would be located far away from the reacting C- 20 center.

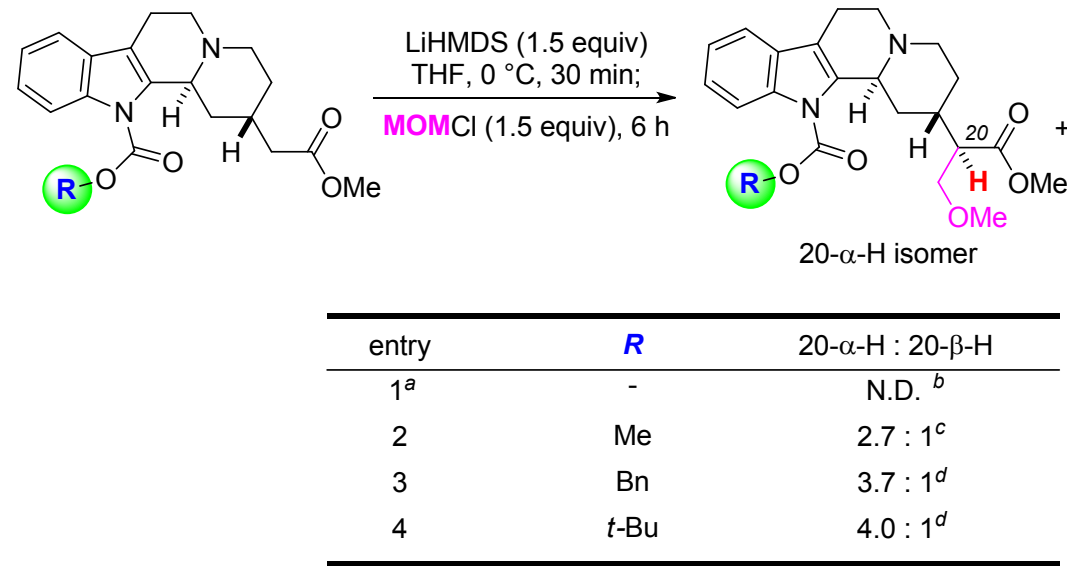

${ }^{a}$ Free Indole compound was used.

${ }^{\mathrm{b}}$ Not determined due to multiple alkylation at both $\mathrm{C}-20$ and indole $\mathrm{N}-\mathrm{H}$ positions.

${ }^{c}$ Two stereoisomers were obtained in an inseparable mixture.

Thus, the facial selectivity was determined by comparison of chemical shift in ${ }^{1} \mathrm{H}$ NMR analysis.

${ }^{\mathrm{d}}$ Determined by isolated isomers.

As demonstrated in the above Scheme, the facial selectivity increased with the size of the R group in the carbamate moiety, which could support our proposed transition state $\mathbf{X}$ for the reactions of enolate derived from $\mathbf{2 3}$ with an electrophile as a more plausible transition state.

\section{3-2. Determination of C-20 Stereochemistry of Alkylation Products}

The stereochemistry of the alkylated products could be assigned by the comparison of the chemical shifts of methyl group in the ester and the methyl group in the methoxymethyl group at the C-20 position. For example, the methyl in the ester of 20$\alpha-\mathrm{H}$ isomer (31a) appears at the up field than that of $20-\beta-\mathrm{H}$ isomer (31b). On the other hand, the methyl peak in the methoxymethyl moiety of $20-\alpha-\mathrm{H}$ isomer (31a) is observed in the down field than that of $20-\beta-\mathrm{H}$ isomer (31b) (see the below figure). 


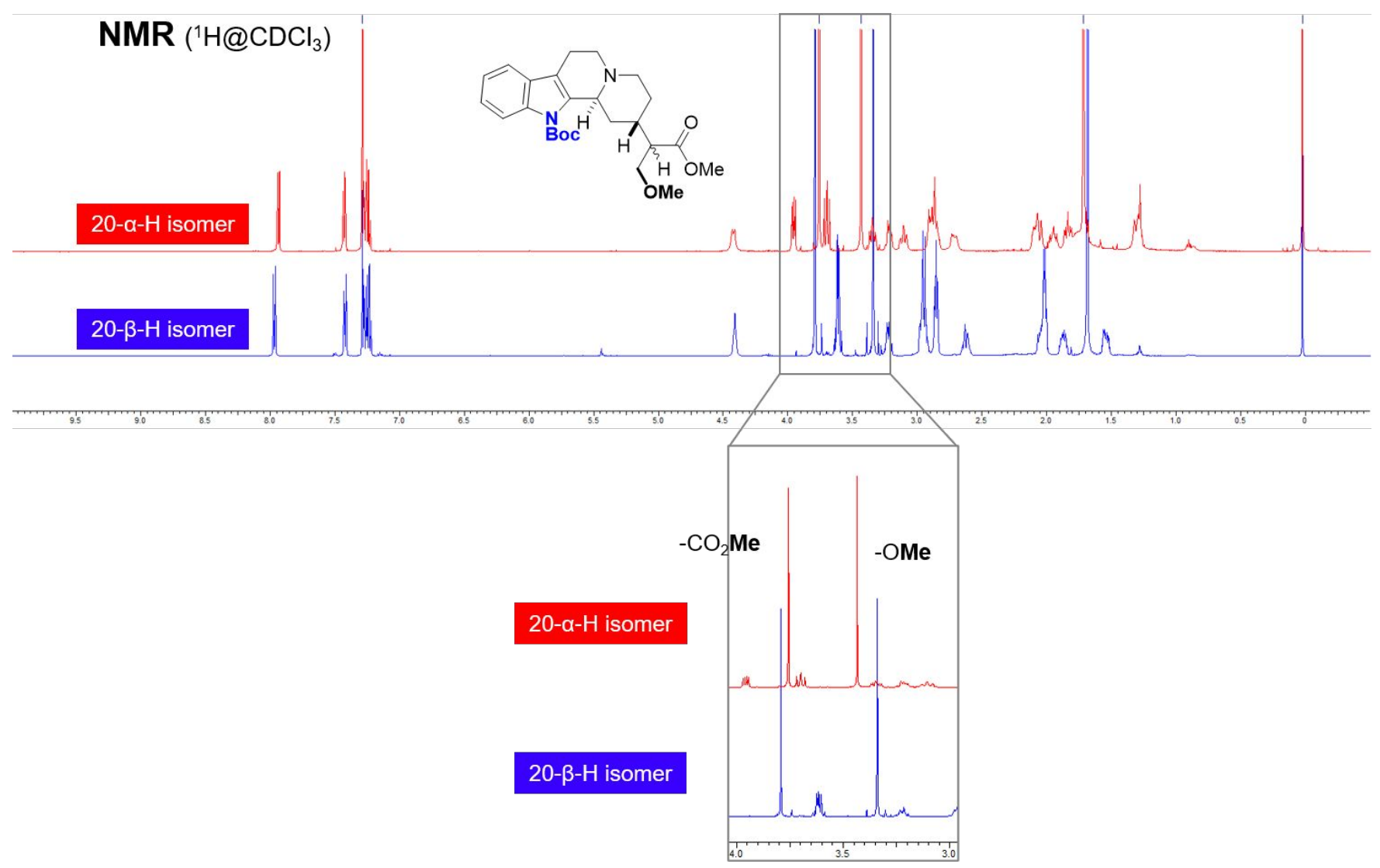

Similar trend in chemical shifts in the ${ }^{1} \mathrm{H}$ NMR spectra were observed in other alkylated products and thus we were able to assign the relative stereochemistry of alkylation products by comparison of chemical shifts of the methyl group in ester moiety and the methyl group in MOM moiety.

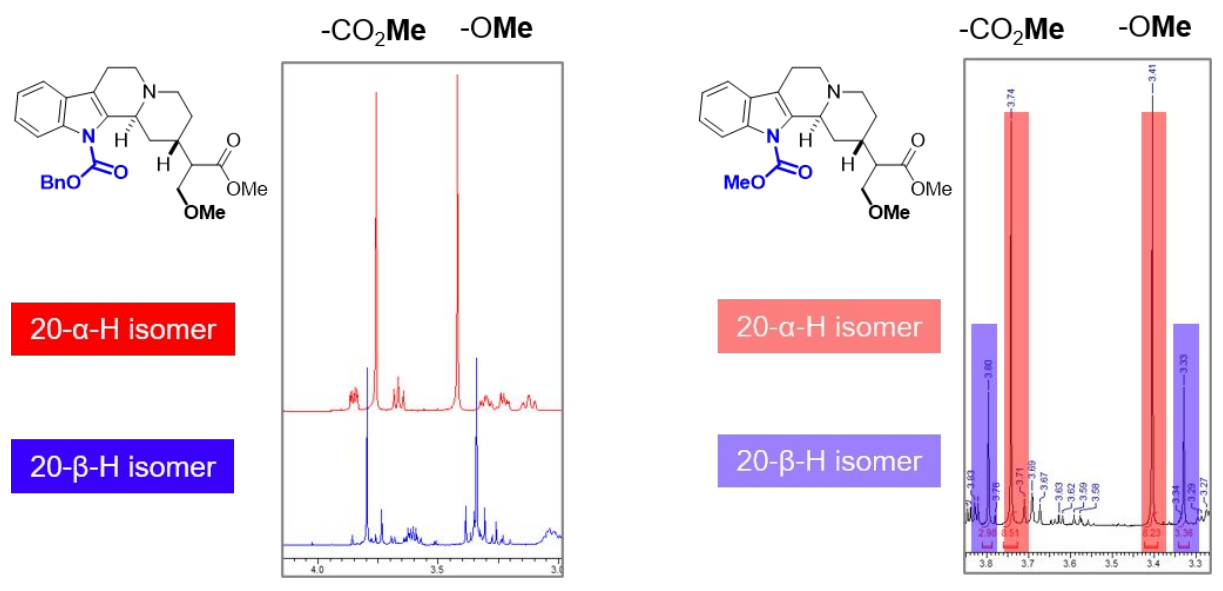




\section{Spectroscopic Data}

4-1. NMR Spectrum of a Crude Product of Ethyl (E)-2-(7,12-Dihydroindolo[2,3-a]quinolizin-2(6H)-ylidene)-3-oxobutanoate (10)

a) ${ }^{1} \mathrm{H}$ NMR Spectrum (in $\mathrm{MeOH}-\mathrm{d}_{4}, 500 \mathrm{MHz}$ )

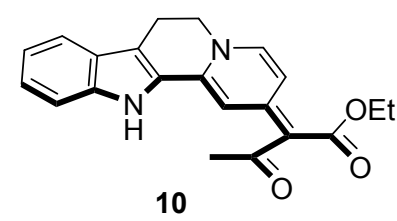

10
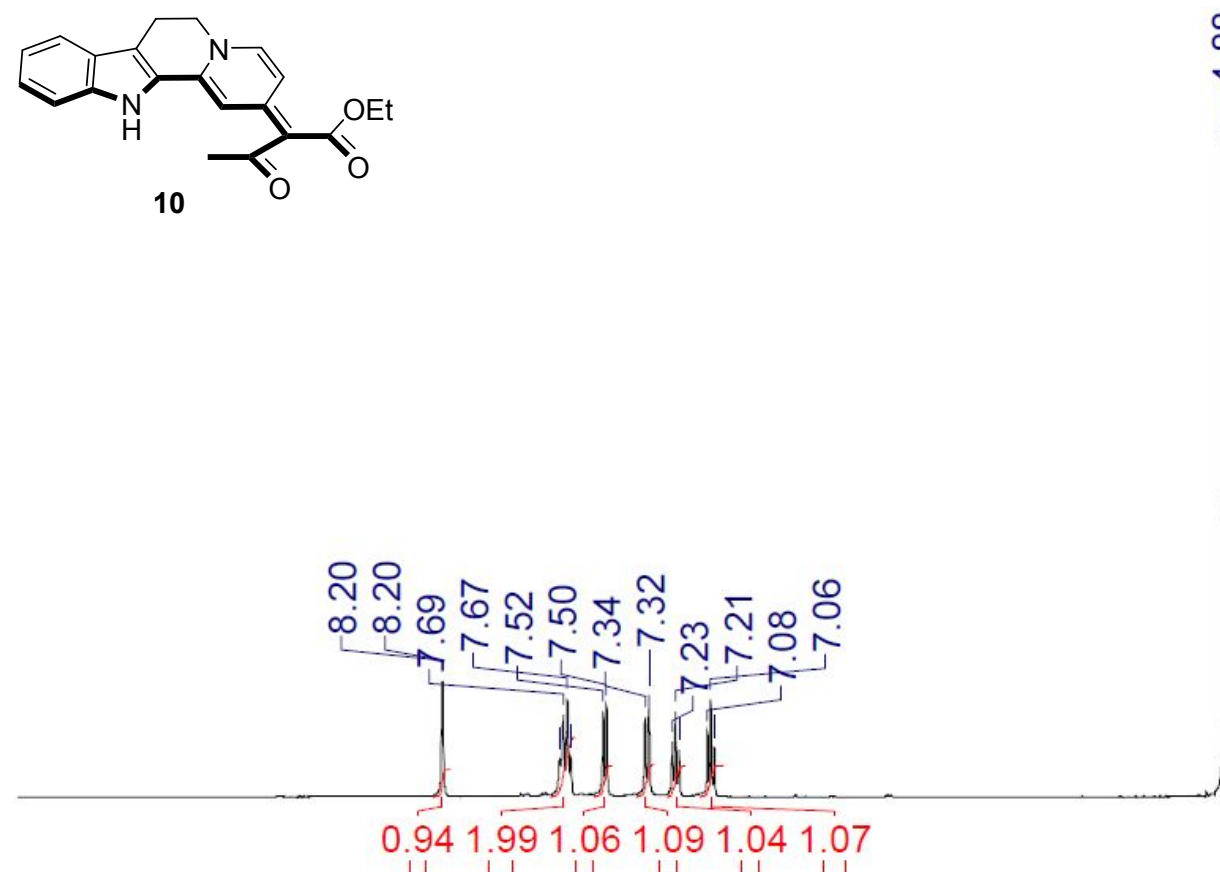

๓

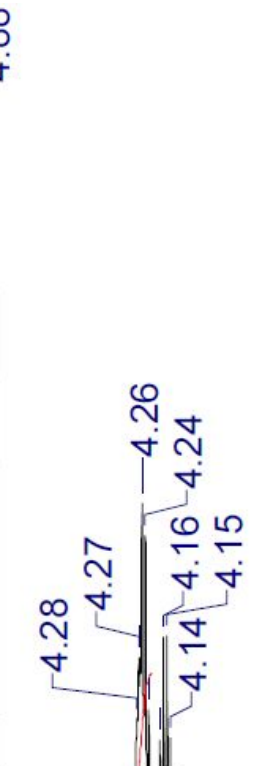

Nิ

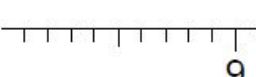

9

8

1
7

6
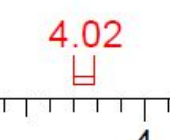

4 
4-2. NMR Spectrum of a Crude Product of 2-(1-Ethoxy-1,3-dioxobutan-2-yl)-7,12-dihydro-6H-indolo[2,3-a]quinolizin-5-ium Trifluoromethanesulfonate (11)

a) ${ }^{1} \mathrm{H}$ NMR Spectrum (in $\mathrm{CDCl}_{3}, 500 \mathrm{MHz}$ )

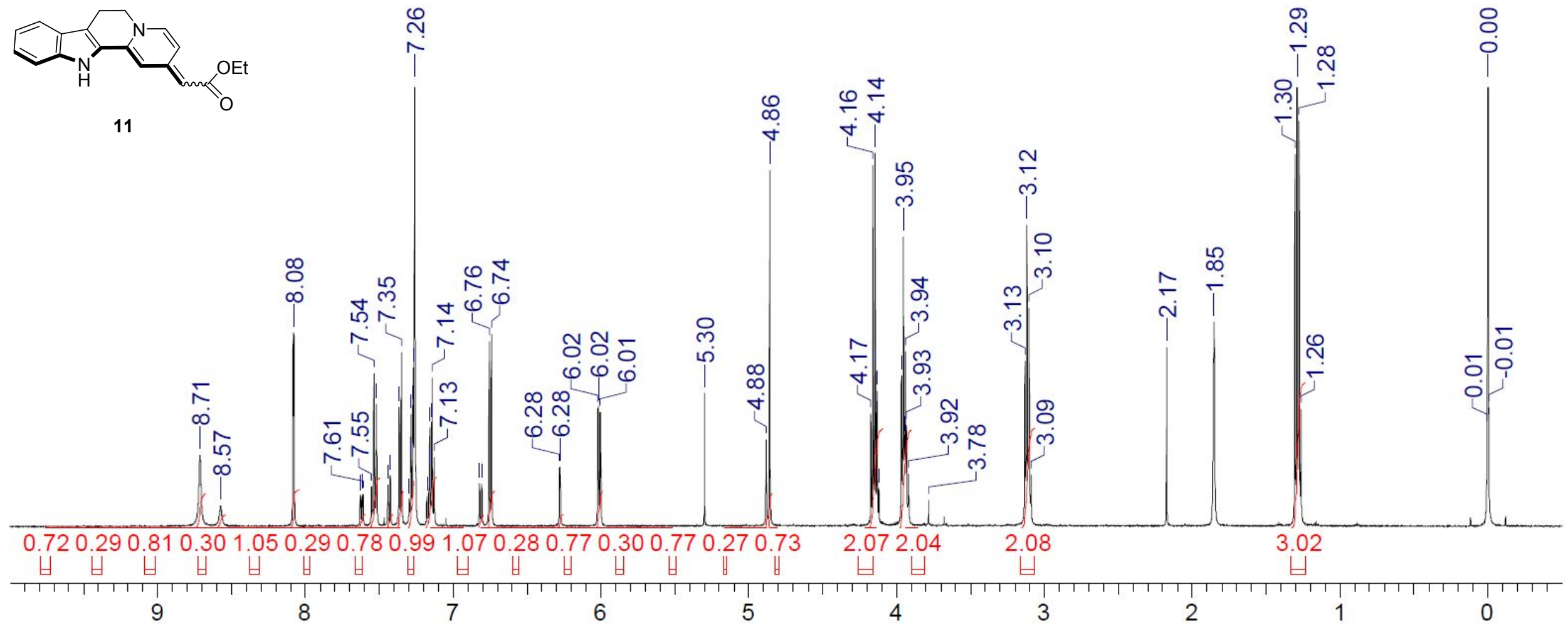


4-3. NMR Spectra of Dimethyl 2-(7,12-Dihydroindolo[2,3-a]quinolizin-2(6H)-ylidene)malonate (15)

a) ${ }^{1} \mathrm{H}$ NMR Spectrum (in $\mathrm{CDCl}_{3}, 500 \mathrm{MHz}$ )

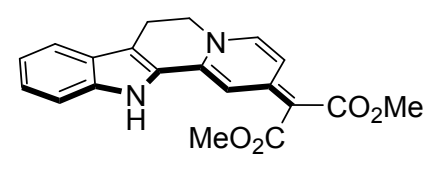

15

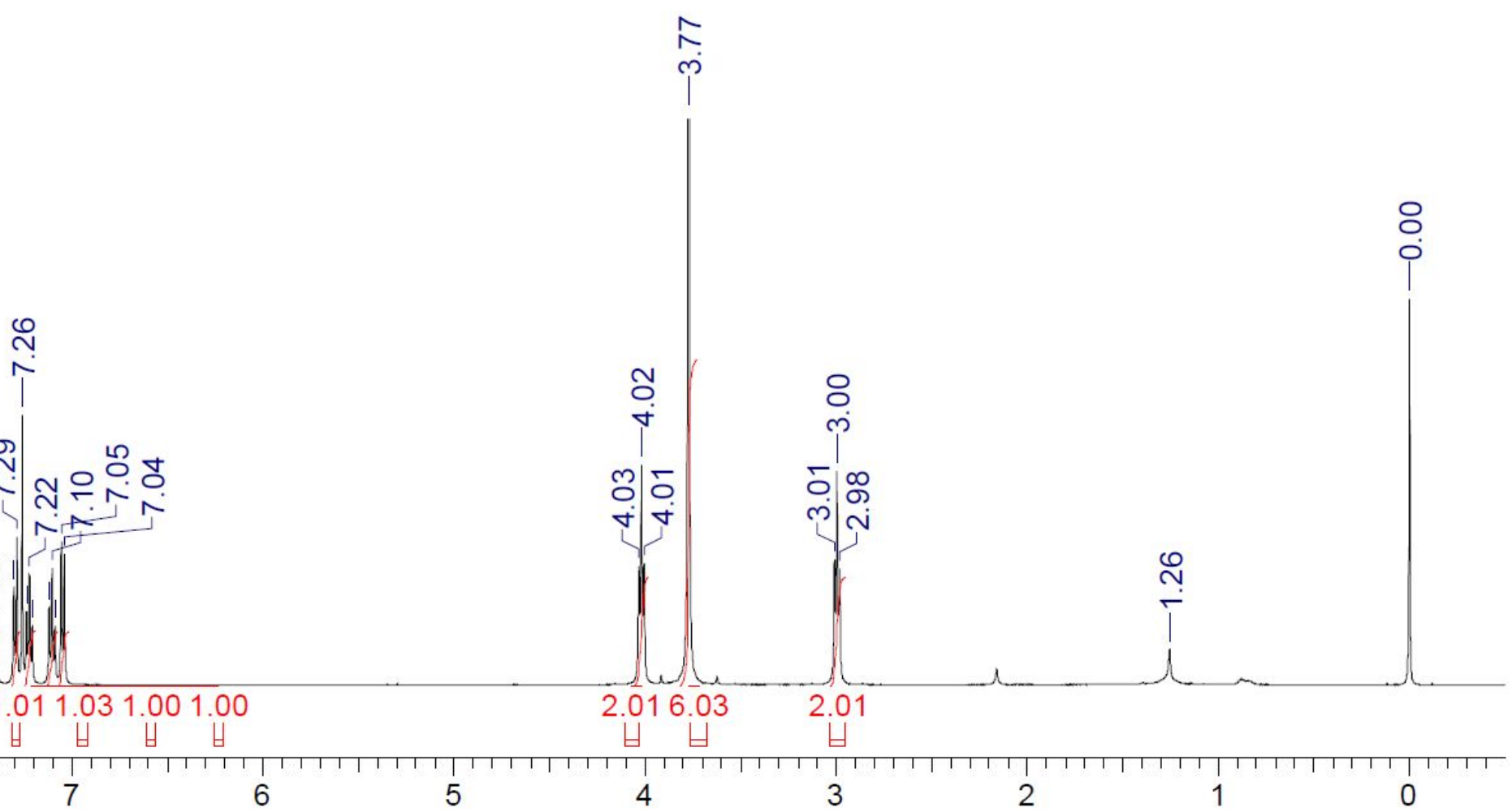


b) ${ }^{13} \mathrm{C}\left\{{ }^{1} \mathrm{H}\right\}$ NMR Spectrum (in $\mathrm{CDCl}_{3}, 125 \mathrm{MHz}$ )

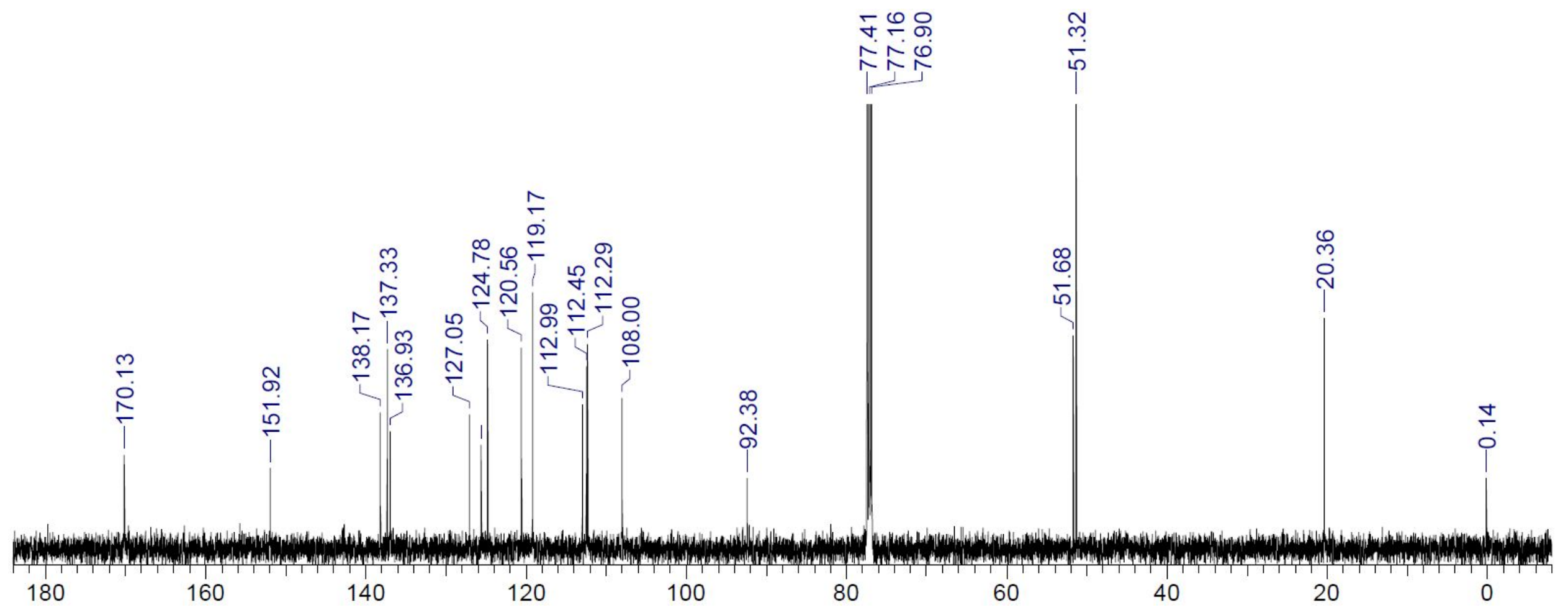


4-4. NMR Spectrum of Dimethyl 2-(1,2,3,4,6,7,12,12b-Octahydroindolo[2,3-a]quinolizin-2-yl)malonate (16)

a) ${ }^{1} \mathrm{H}$ NMR Spectrum (in $\mathrm{CDCl}_{3}, 500 \mathrm{MHz}$ )

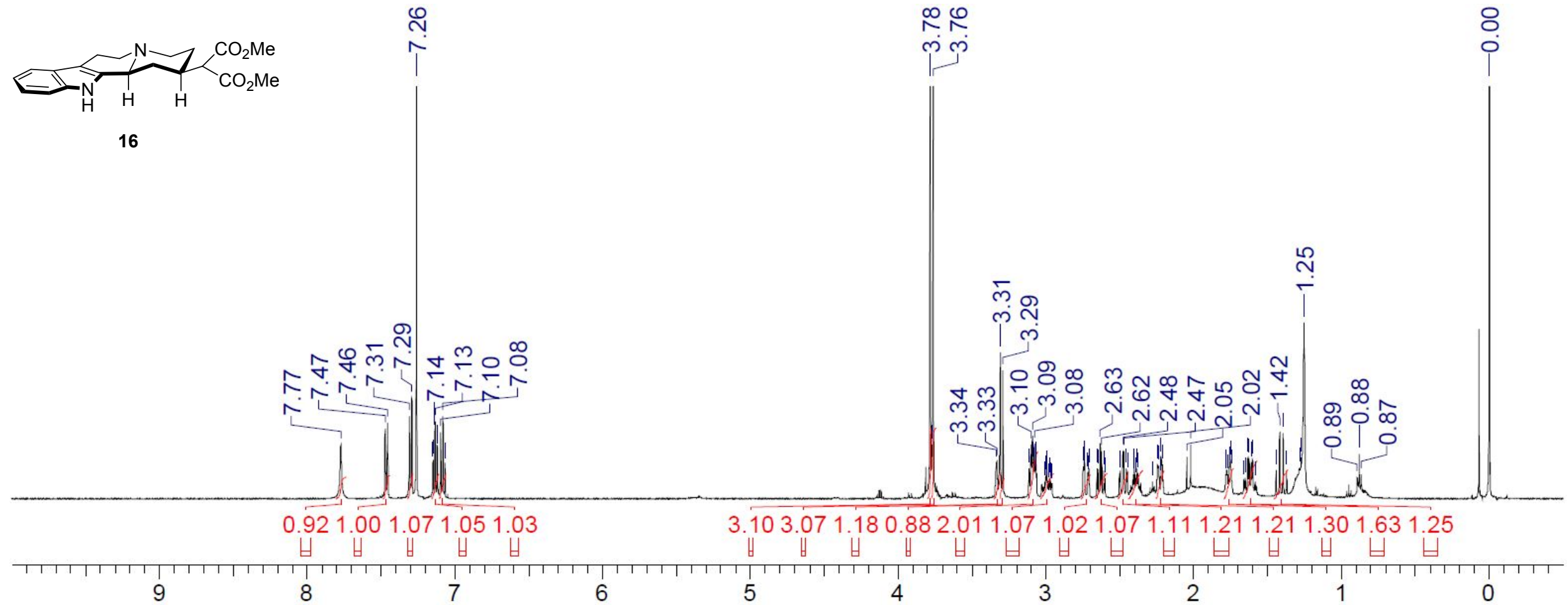


4-5. NMR Spectra of Dimethyl 2-(12-(tert-Butoxycarbonyl)-7,12-dihydroindolo[2,3-a]quinolizin-2(6H)-ylidene)malonate (17)

a) ${ }^{1} \mathrm{H}$ NMR Spectrum (in $\mathrm{CDCl}_{3}, 500 \mathrm{MHz}$ )

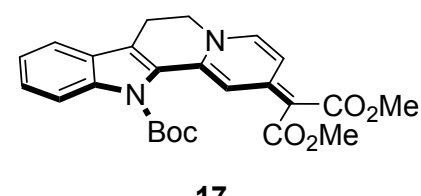

17

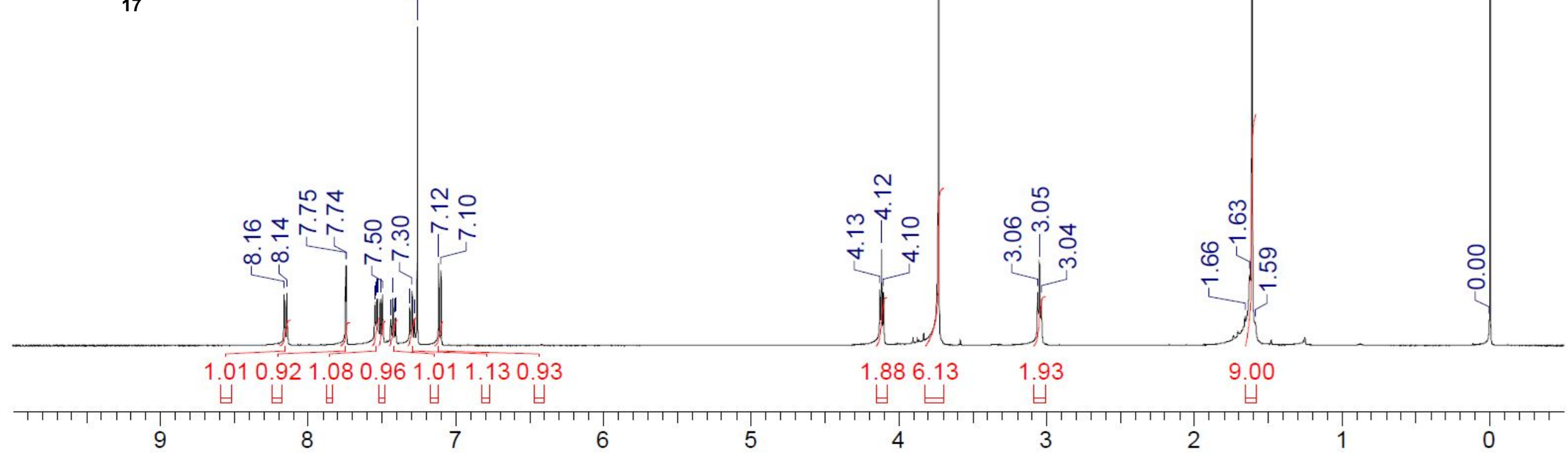


b) ${ }^{13} \mathrm{C}\left\{{ }^{1} \mathrm{H}\right\}$ NMR Spectrum (in $\mathrm{CDCl}_{3}, 125 \mathrm{MHz}$ )

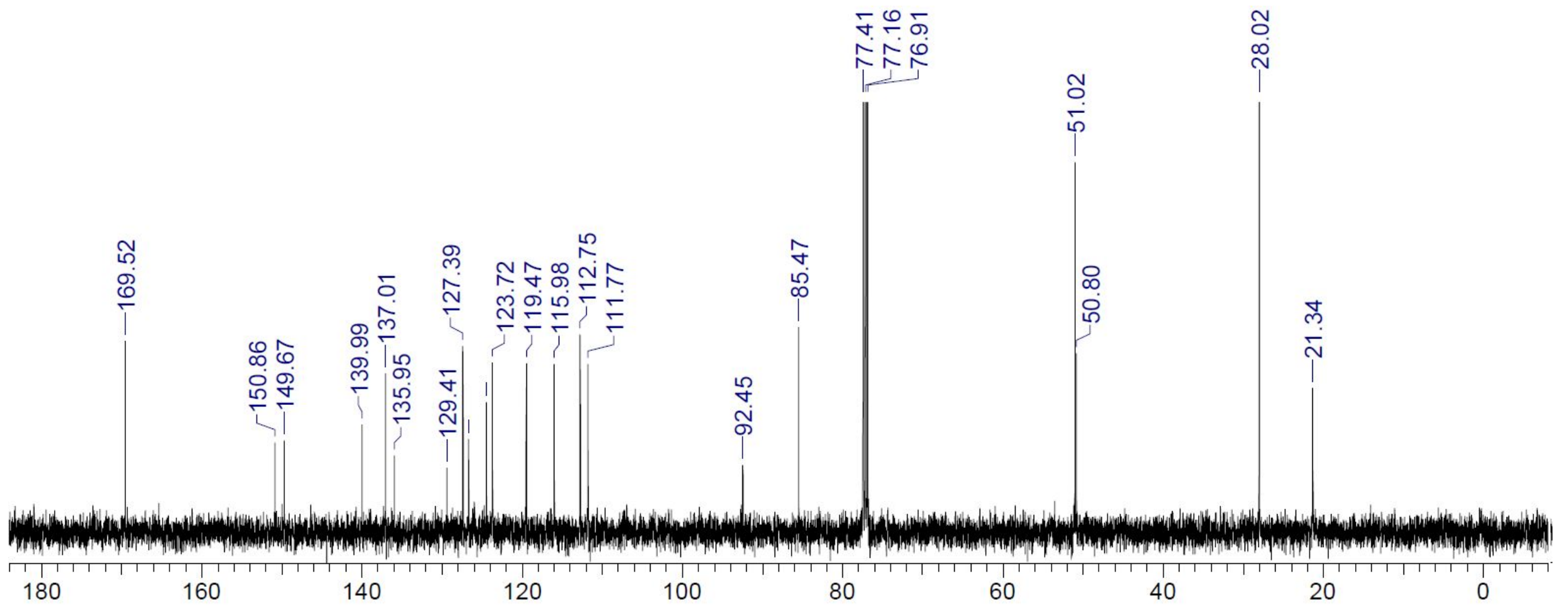


4-6. NMR Spectrum of Dimethyl 2-(1,2,3,4,6,7,12,12b-Octahydroindolo[2,3-a]quinolizin-2-yl)malonate (18)

a) ${ }^{1} \mathrm{H}$ NMR Spectrum (in $\mathrm{CDCl}_{3}, 500 \mathrm{MHz}$ )

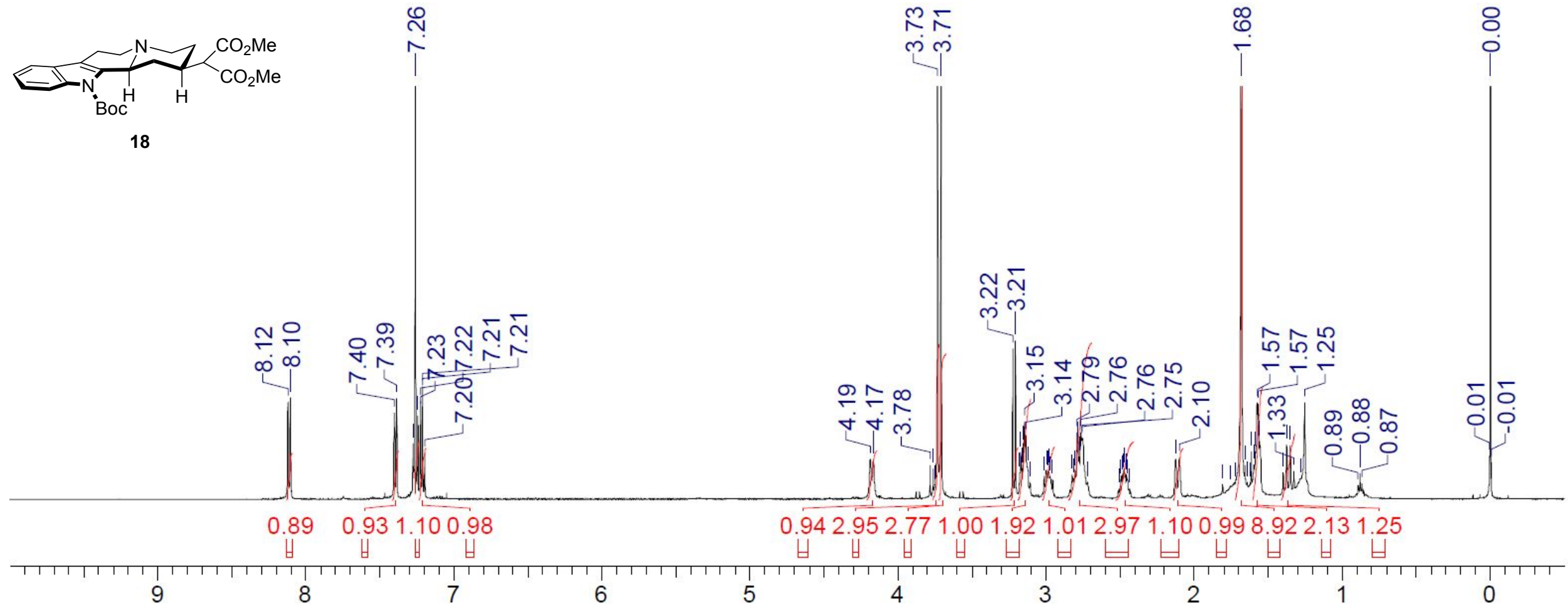


4-7. NMR Spectrum of Dimethyl 2-(1,2,3,4,6,7,12,12b-Octahydroindolo[2,3-a]quinolizin-2-yl)malonate (15-epi-18)

a) ${ }^{1} \mathrm{H}$ NMR Spectrum (in $\mathrm{CDCl}_{3}, 500 \mathrm{MHz}$ )

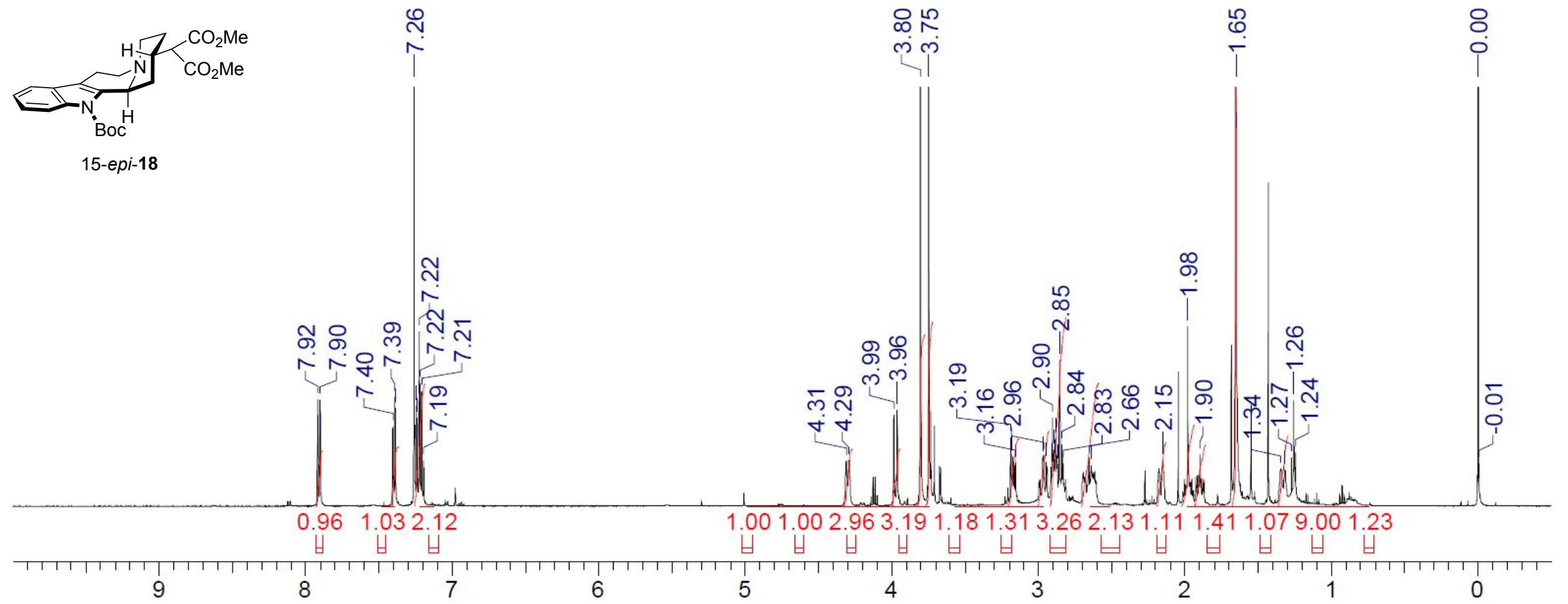


4-8. NMR Spectra of Dimethyl 2-(1,4,6,7,12,12b-Hexahydroindolo[2,3-a]quinolizin-2-yl)malonate (19)

a) ${ }^{1} \mathrm{H}$ NMR Spectrum (in $\mathrm{CDCl}_{3}, 500 \mathrm{MHz}$ )
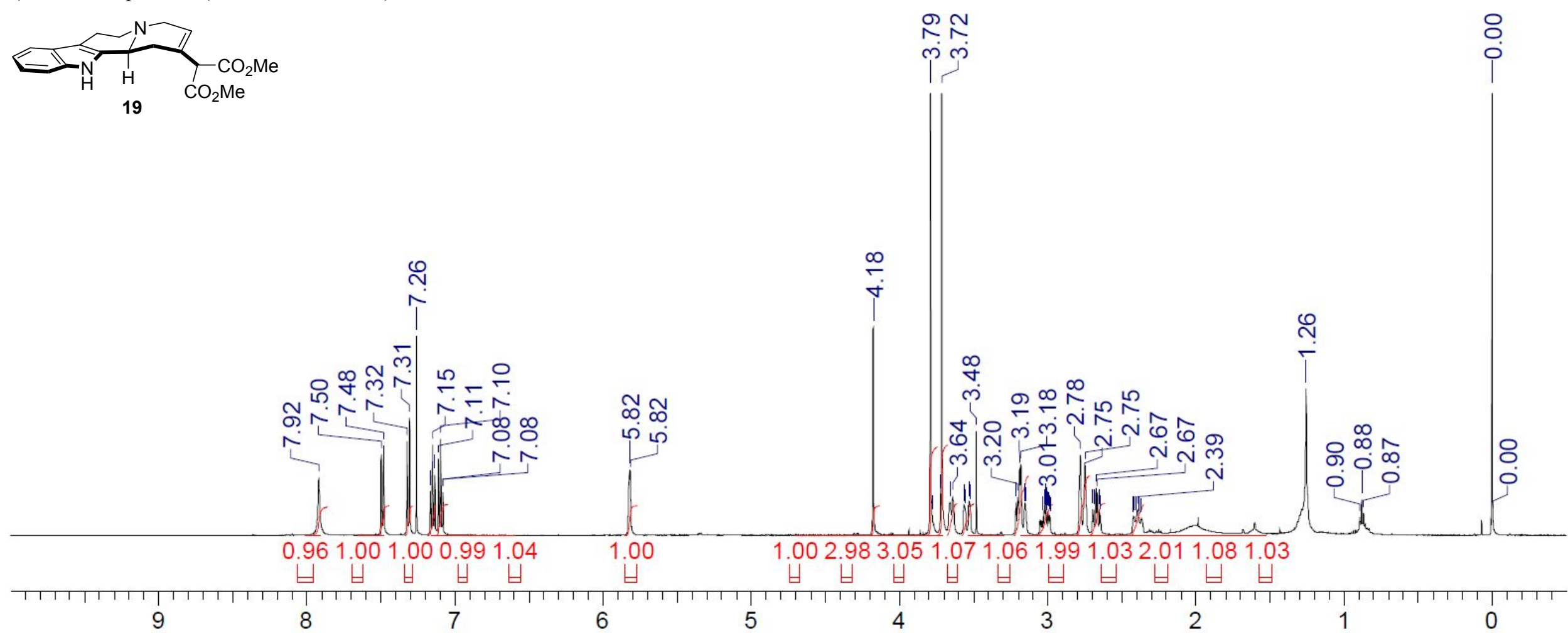
b) ${ }^{13} \mathrm{C}\left\{{ }^{1} \mathrm{H}\right\}$ NMR Spectrum (in $\mathrm{CDCl}_{3}, 125 \mathrm{MHz}$ )

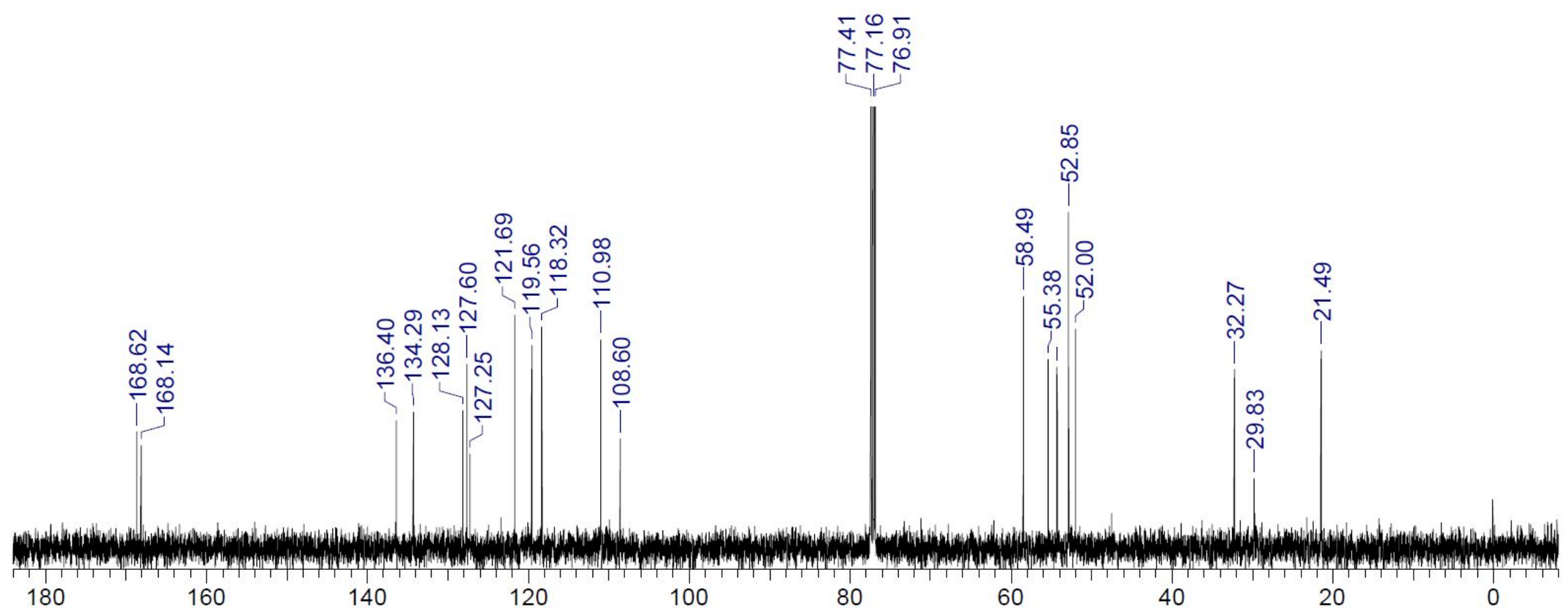


4-9. NMR Spectrum of Methyl 2-(1,4,6,7,12,12b-Hexahydroindolo[2,3-a]quinolizin-2-yl)acetate (21)

a) ${ }^{1} \mathrm{H}$ NMR Spectrum (in $\mathrm{CDCl}_{3}, 500 \mathrm{MHz}$ )

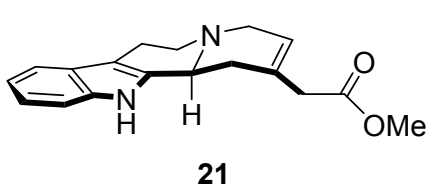

21

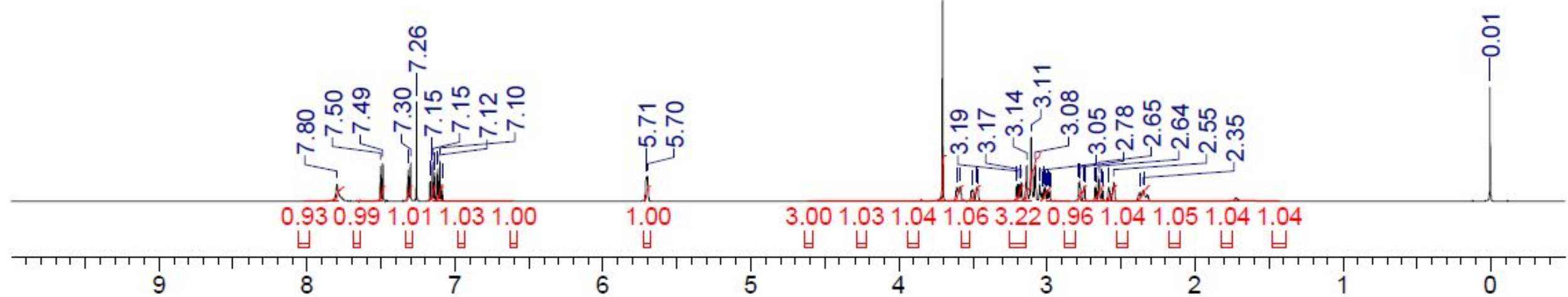


4-10. NMR Spectrum of tert-Butyl 2-(2-Methoxy-2-oxoethyl)-1,6,7,12b-tetrahydroindolo[2,3-a]quinolizine-12(4H)-carboxylate (22)

a) ${ }^{1} \mathrm{H}$ NMR Spectrum (in $\mathrm{CDCl}_{3}, 500 \mathrm{MHz}$ )
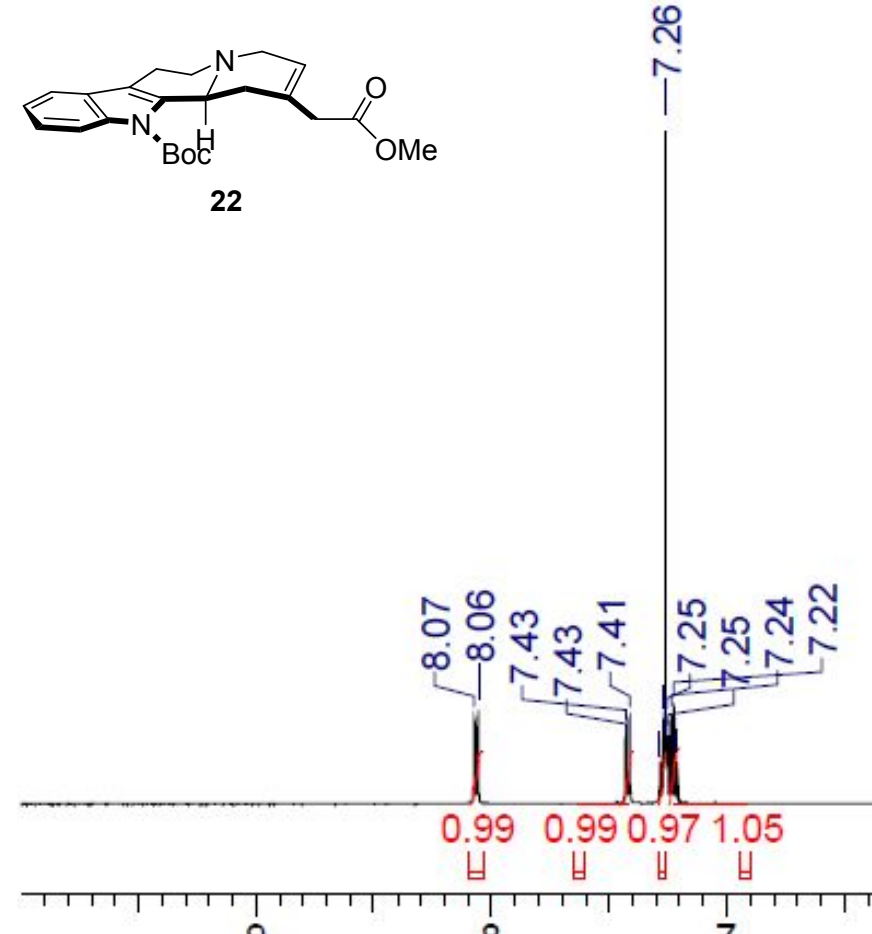

$\stackrel{\sim}{\sim}$

\section{OMe}

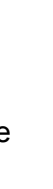

(1)

$\hat{\imath}$

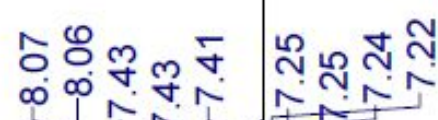

8

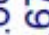

L

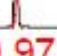

U.97

1110111 5 $\hat{0}$

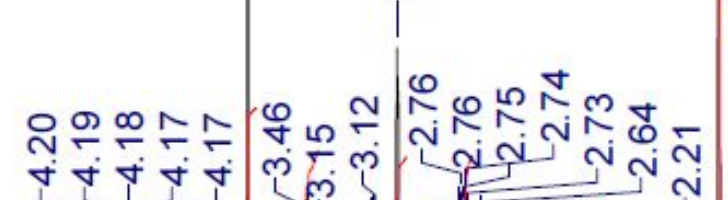

8
1.052 .922 .141 .032 .021 .122 .051 .101 .109 .00

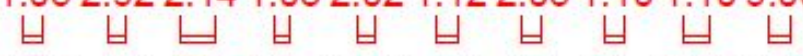

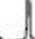

3 (1T)

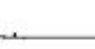


4-11. NMR Spectrum of 2,12b-trans-tert-Butyl 2-(2-Methoxy-2-oxoethyl)-1,3,4,6,7,12b-hexahydroindolo[2,3-a]quinolizine-12(2H)-carboxylate (23)

a) ${ }^{1} \mathrm{H}$ NMR Spectrum (in $\mathrm{CDCl}_{3}, 500 \mathrm{MHz}$ )

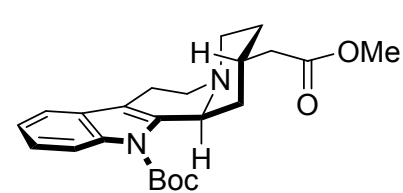

23

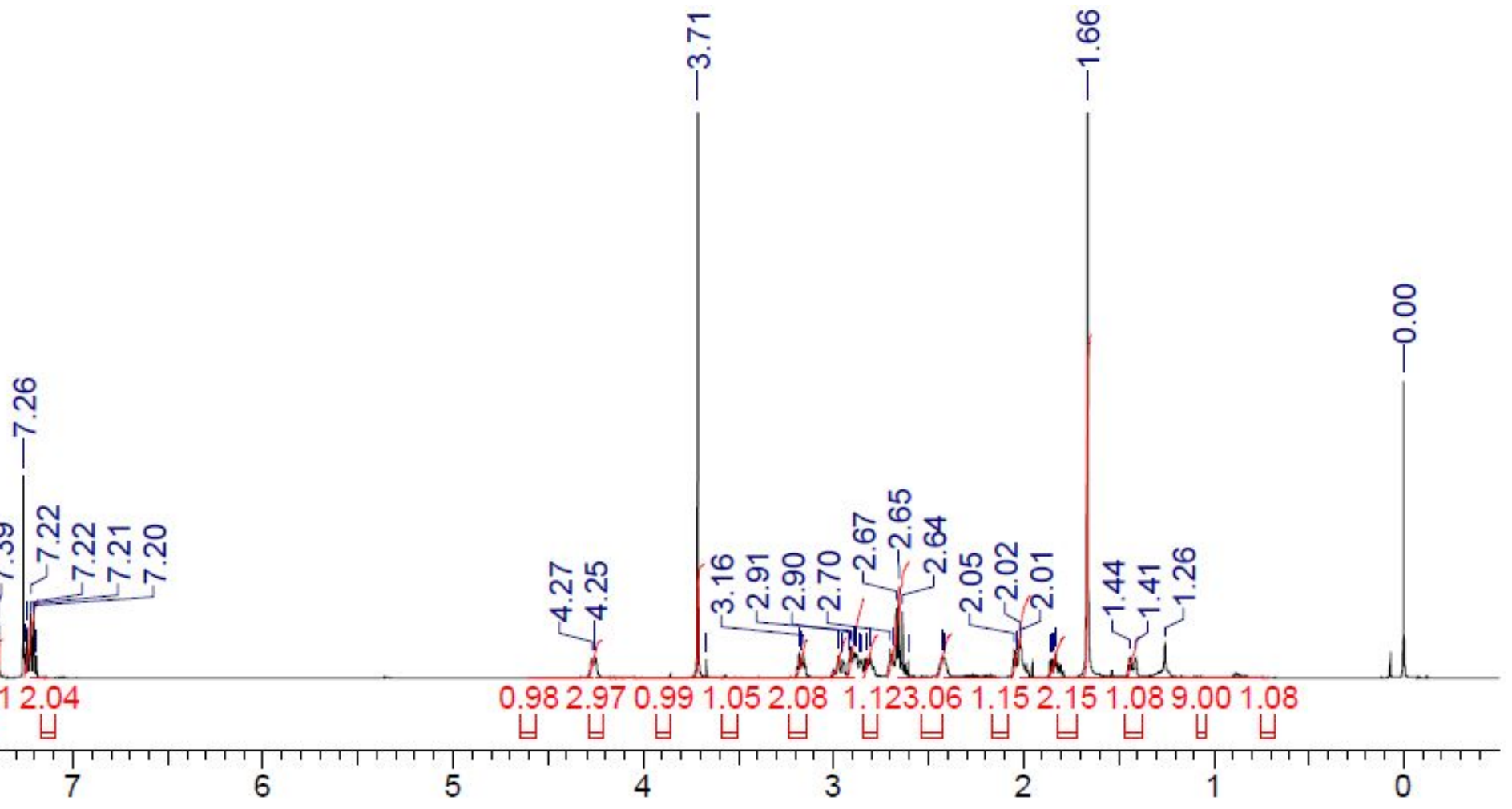


4-12. NMR Spectrum of 2,12b-cis-tert-Butyl 2-(2-Methoxy-2-oxoethyl)-1,3,4,6,7,12b-hexahydroindolo[2,3-a]quinolizine-12(2H)-carboxylate (15-epi-23)

a) ${ }^{1} \mathrm{H}$ NMR Spectrum (in $\mathrm{CDCl}_{3}, 500 \mathrm{MHz}$ )

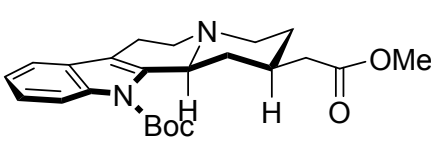

15-ері-23

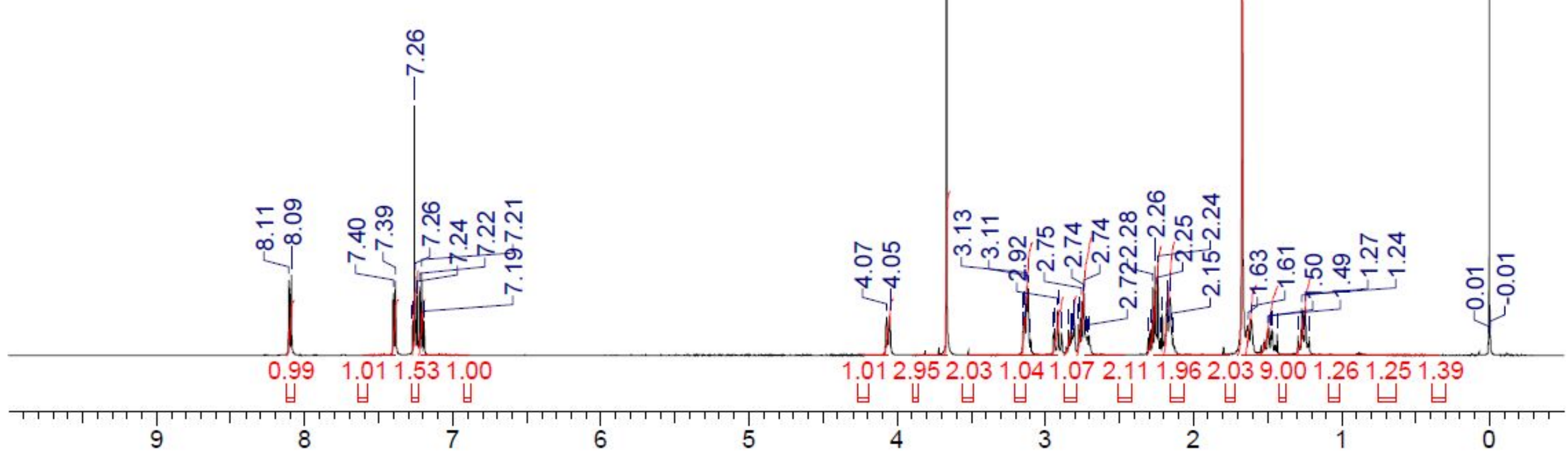


4-13. NMR Spectrum of tert-Butyl 2-(1-Methoxy-1-oxo-4-(phenylsulfinyl)butan-2-yl)-1,3,4,6,7,12b-hexahydroindolo[2,3-a]quinolizine-12(2H)-carboxylate (24)

a) ${ }^{1} \mathrm{H}$ NMR Spectrum (in $\mathrm{CDCl}_{3}, 500 \mathrm{MHz}$ )
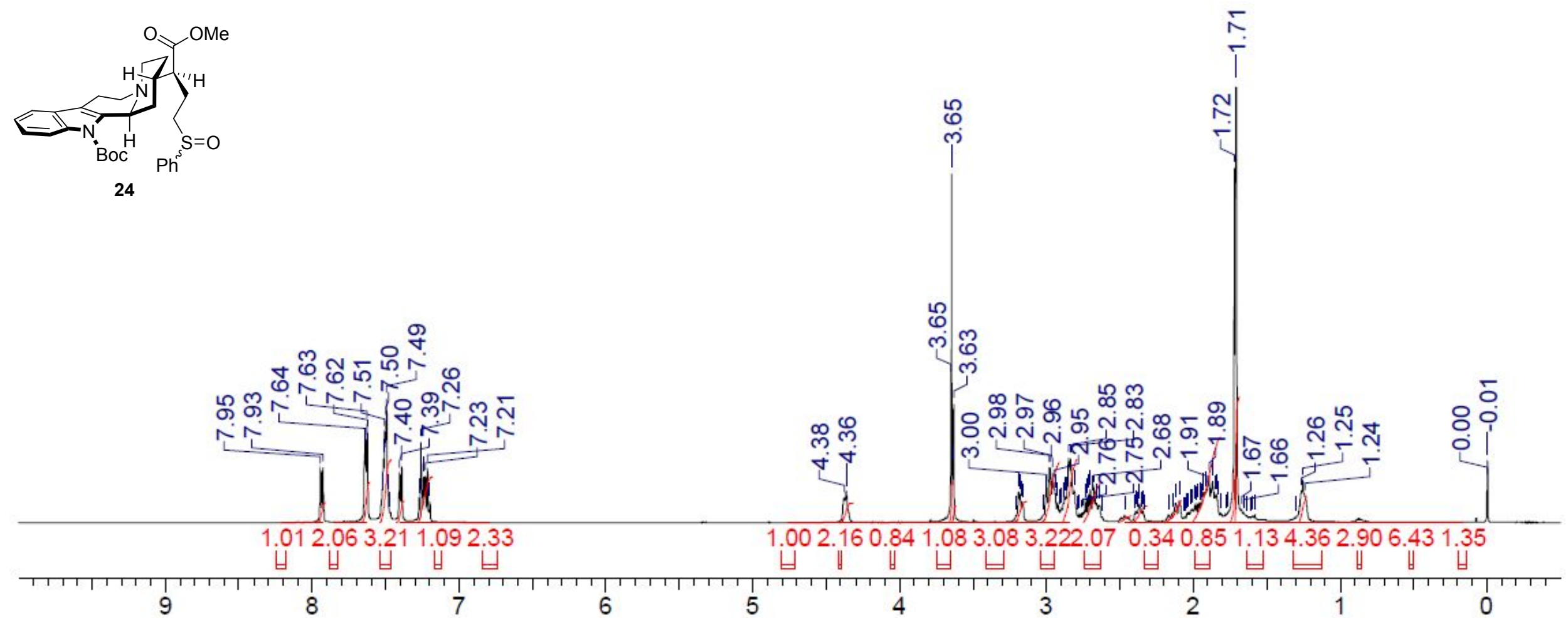
4-14. NMR Spectrum of 2-(1,2,3,4,6,7,12,12b-Octahydroindolo[2,3-a]quinolizin-2-yl)-4-(phenylsulfinyl)butan-1-ol (25)

a) ${ }^{1} \mathrm{H}$ NMR Spectrum of a Crude Product 25 (in $\mathrm{CDCl}_{3}, 500 \mathrm{MHz}$ )

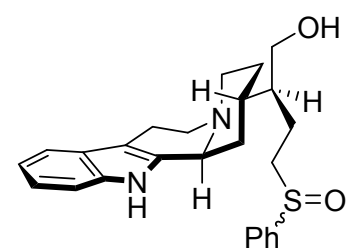

25

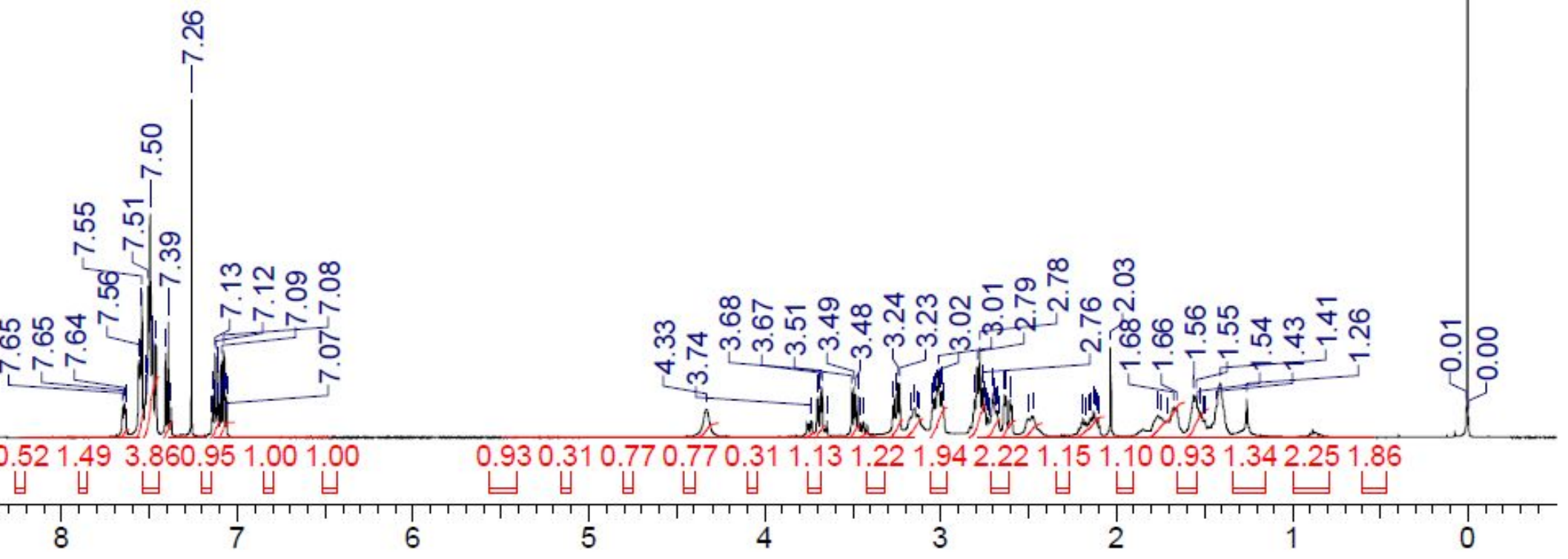


4-15. NMR Spectra of 20-epi-Antirhine (2)

a) ${ }^{1} \mathrm{H}$ NMR Spectrum (in $\mathrm{CDCl}_{3}, 500 \mathrm{MHz}$ )

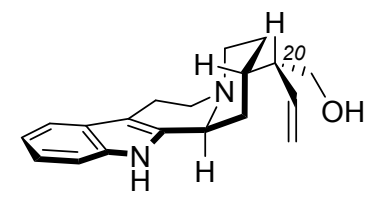

20-epi-antirhine $(2)$

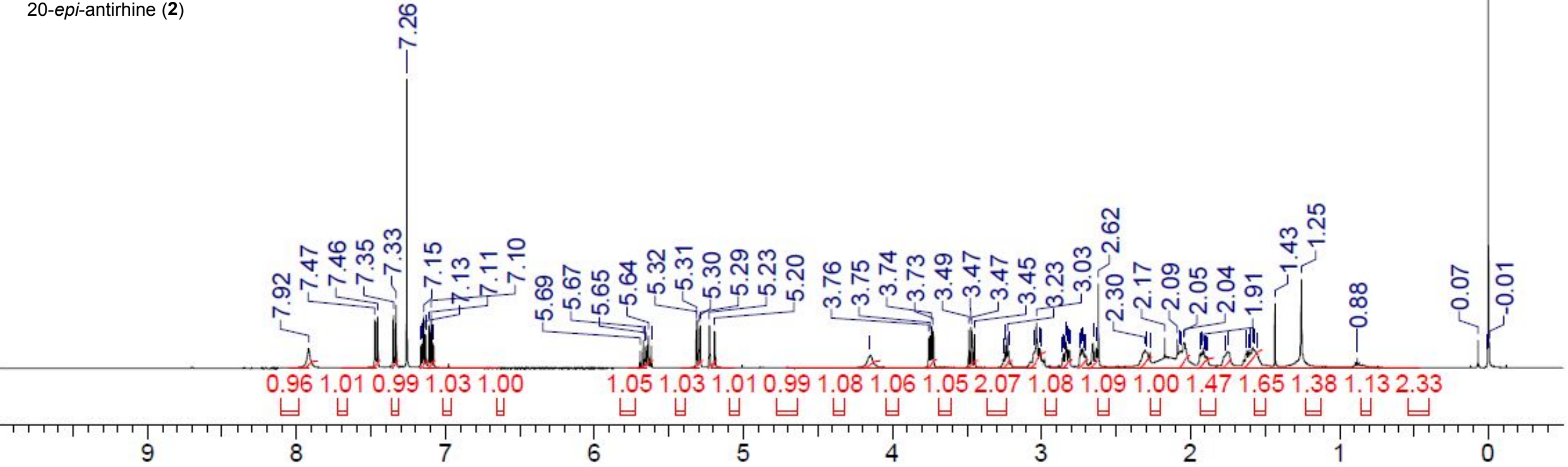


b) ${ }^{13} \mathrm{C}\left\{{ }^{1} \mathrm{H}\right\}$ NMR Spectrum (in $\mathrm{CDCl}_{3}, 125 \mathrm{MHz}$ )

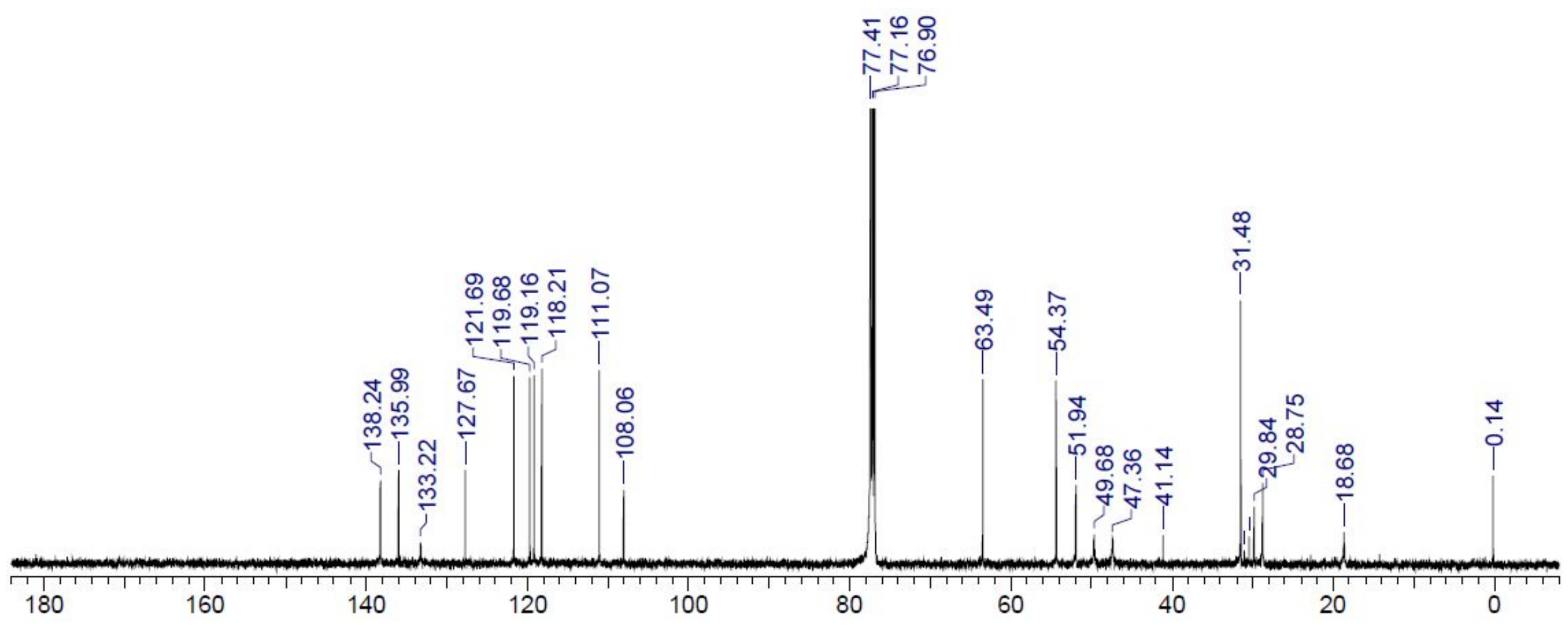


4-16. NMR Spectra of 20-epi-18,19-Dihydroantirhine (2-H $)$

a) ${ }^{1} \mathrm{H}$ NMR Spectrum (in $\mathrm{CDCl}_{3}, 500 \mathrm{MHz}$ )

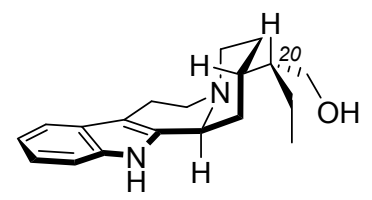

20-epi-18,19-dihydroantirhine $\left(2-\mathrm{H}_{2}\right)$

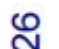

†
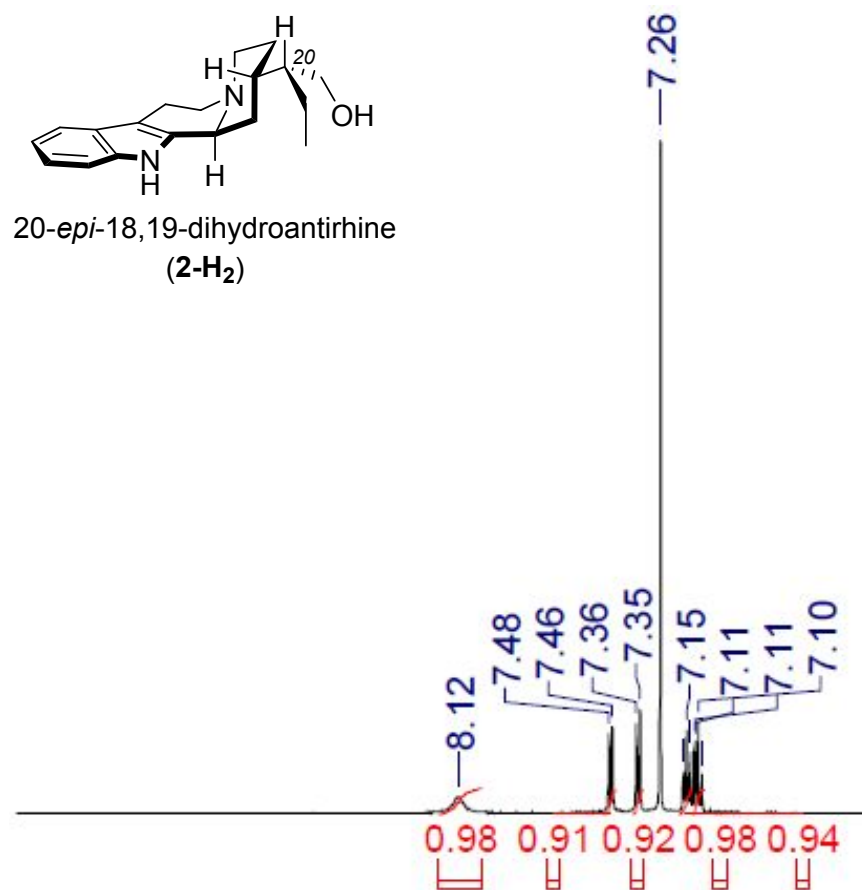

ले

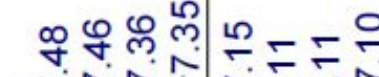

-LiN TN

0.980 .910 .920 .980 .94

$\frac{k}{931.900 .991 .021 .081 .031 .051 .151 .131 .072 .102 .231 .151 .913 .00}$

8

7

5

4

1

0 
b) ${ }^{13} \mathrm{C}\left\{{ }^{1} \mathrm{H}\right\}$ NMR Spectrum (in $\mathrm{CDCl}_{3}, 125 \mathrm{MHz}$ )

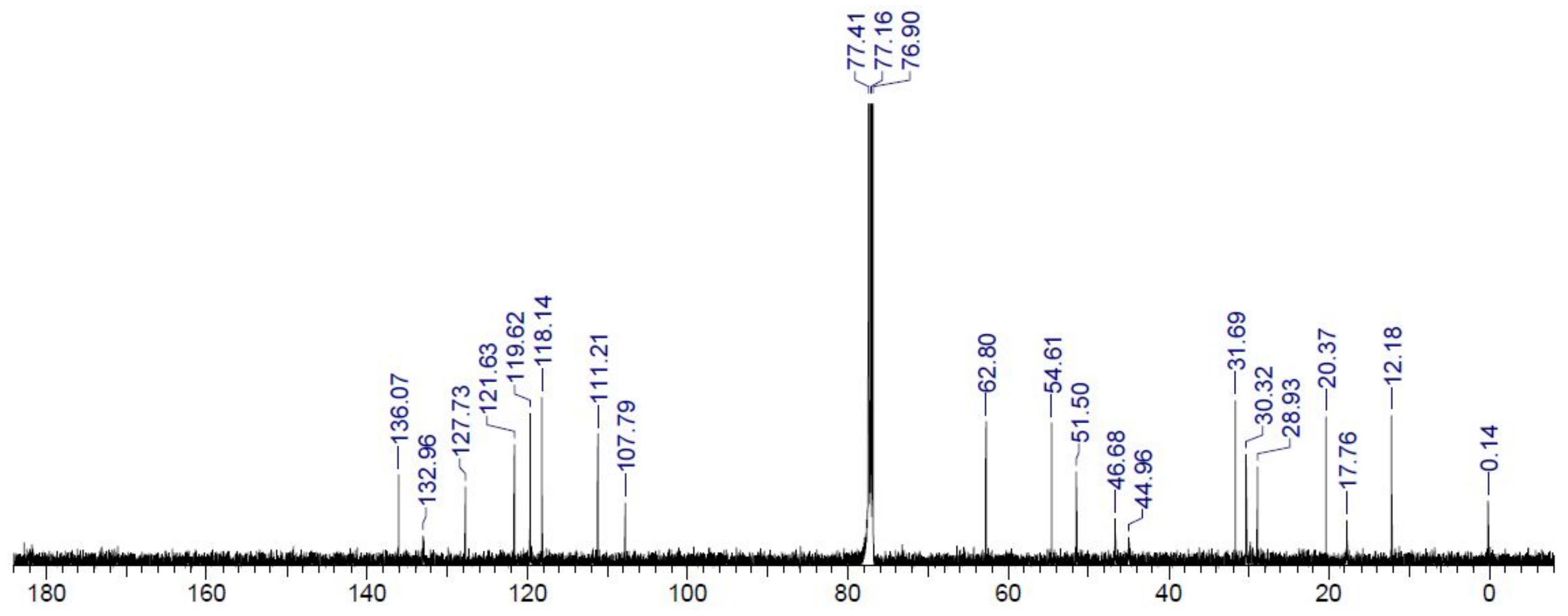


4-17. NMR Spectra of tert-Butyl 2-(2-Oxotetrahydrofuran-3-yl)-1,3,4,6,7,12b-hexahydroindolo[2,3-a]quinolizine-12(2H)-carboxylate (27)

a) ${ }^{1} \mathrm{H}$ NMR Spectrum (in $\mathrm{CDCl}_{3}, 500 \mathrm{MHz}$ )
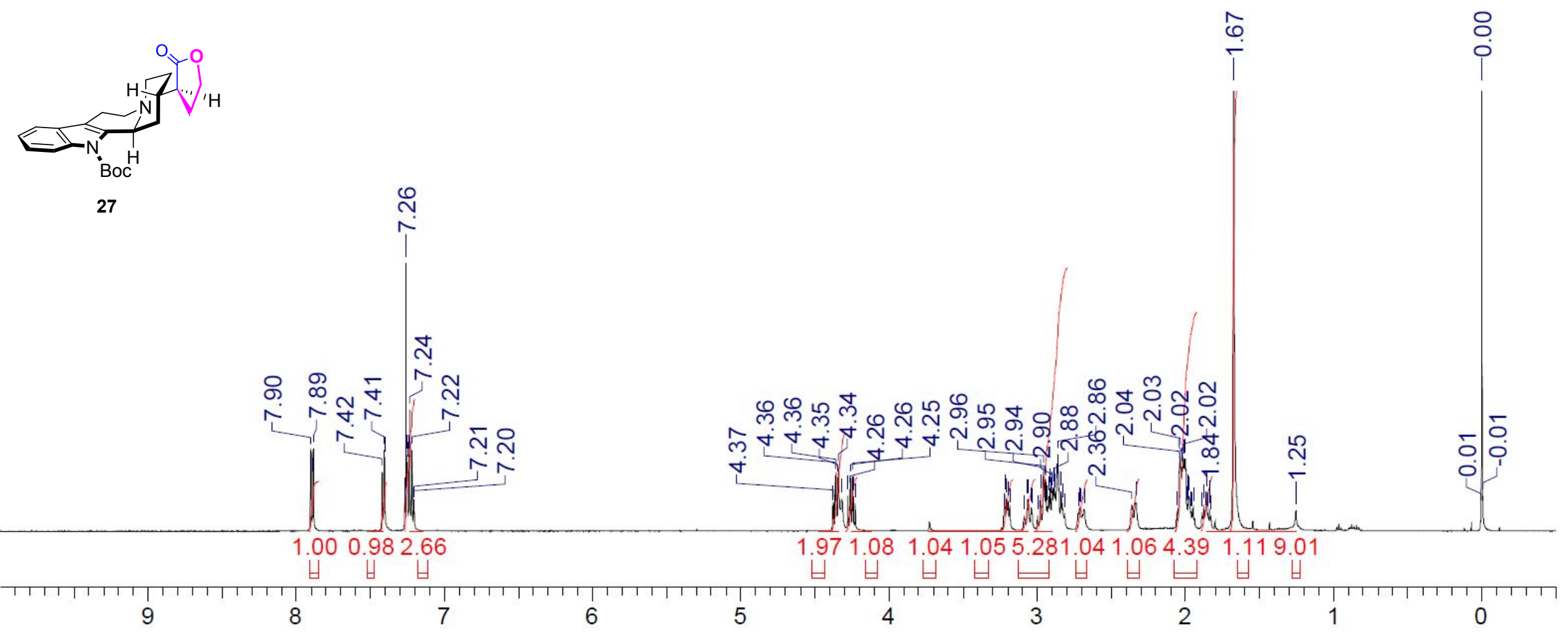
b) ${ }^{13} \mathrm{C}\left\{{ }^{1} \mathrm{H}\right\}$ NMR Spectrum (in $\mathrm{CDCl}_{3}, 125 \mathrm{MHz}$ )

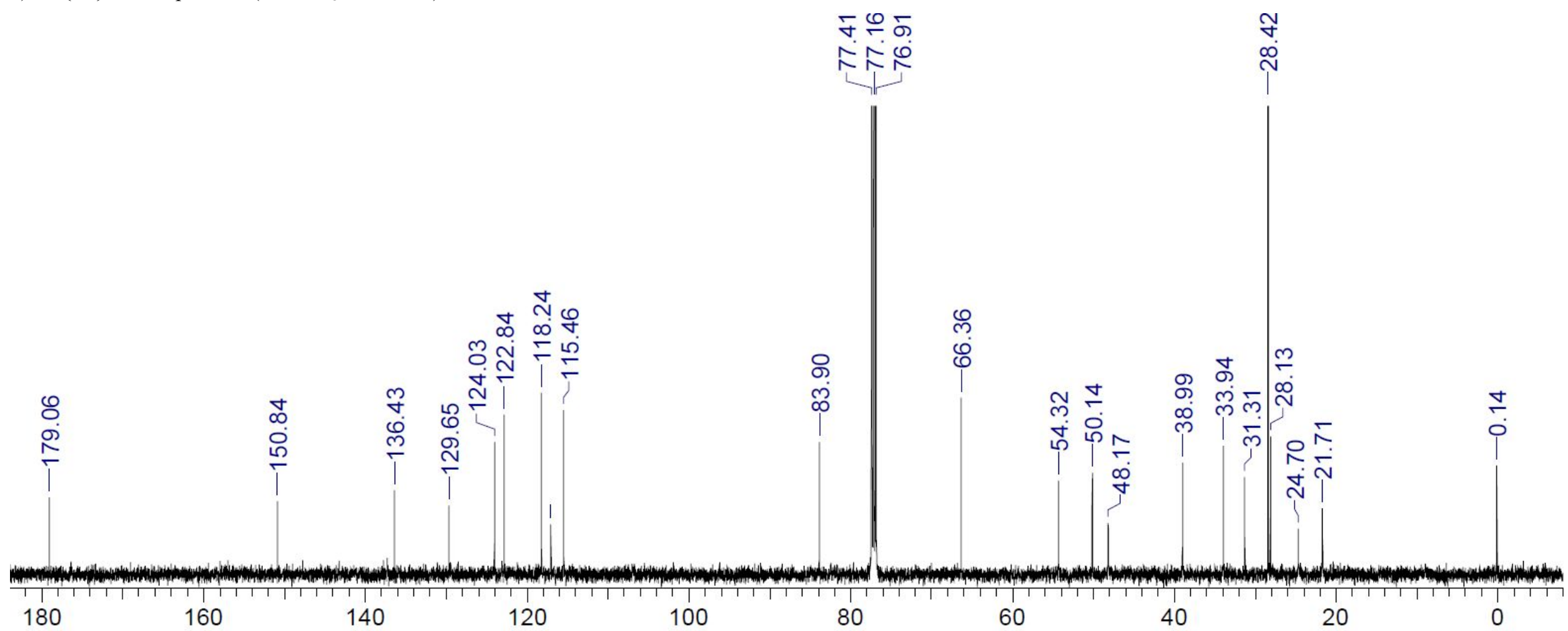


4-18. NMR Spectra of tert-Butyl 2-(2-Oxotetrahydrofuran-3-yl)-1,3,4,6,7,12b-hexahydroindolo[2,3-a]quinolizine-12(2H)-carboxylate (20-epi-27)

a) ${ }^{1} \mathrm{H}$ NMR Spectrum (in $\mathrm{CDCl}_{3}, 500 \mathrm{MHz}$ )

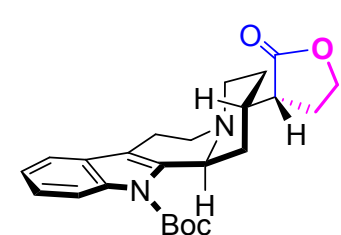

20-ері-27

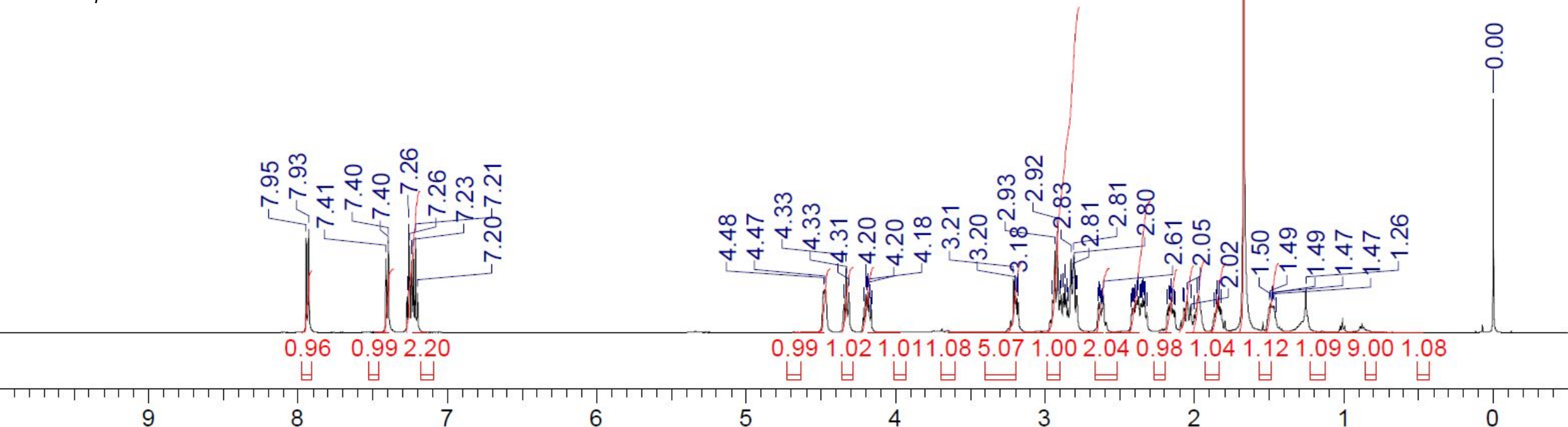


b) ${ }^{13} \mathrm{C}\left\{{ }^{1} \mathrm{H}\right\}$ NMR Spectrum (in $\mathrm{CDCl}_{3}, 125 \mathrm{MHz}$ )

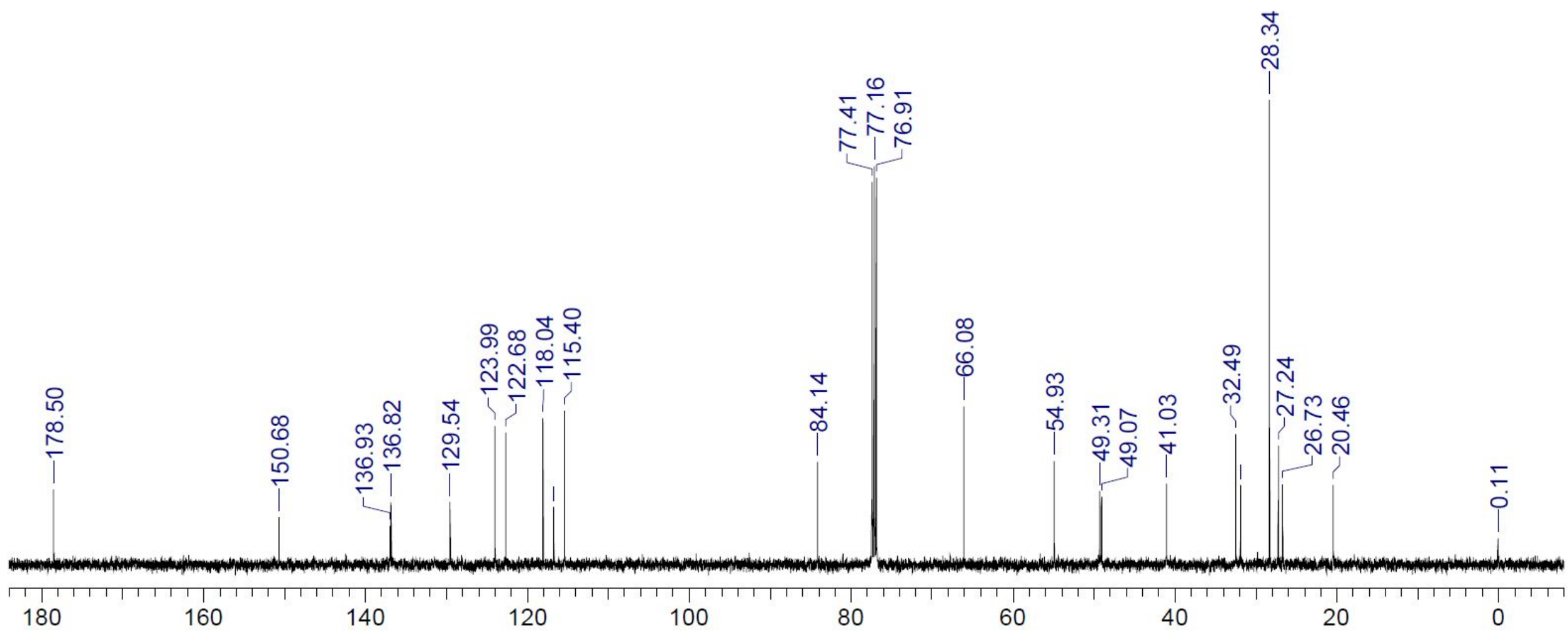


4-19. NMR Spectrum of 2-(1,2,3,4,6,7,12,12b-Octahydroindolo[2,3-a]quinolizin-2-yl)butane-1,4-diol (28)

a) ${ }^{1} \mathrm{H}$ NMR Spectrum (in $\mathrm{CDCl}_{3}, 500 \mathrm{MHz}$ )

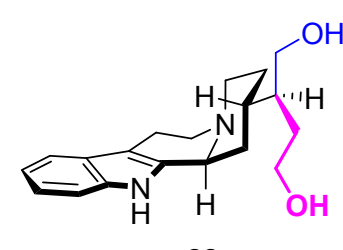

28

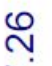

i

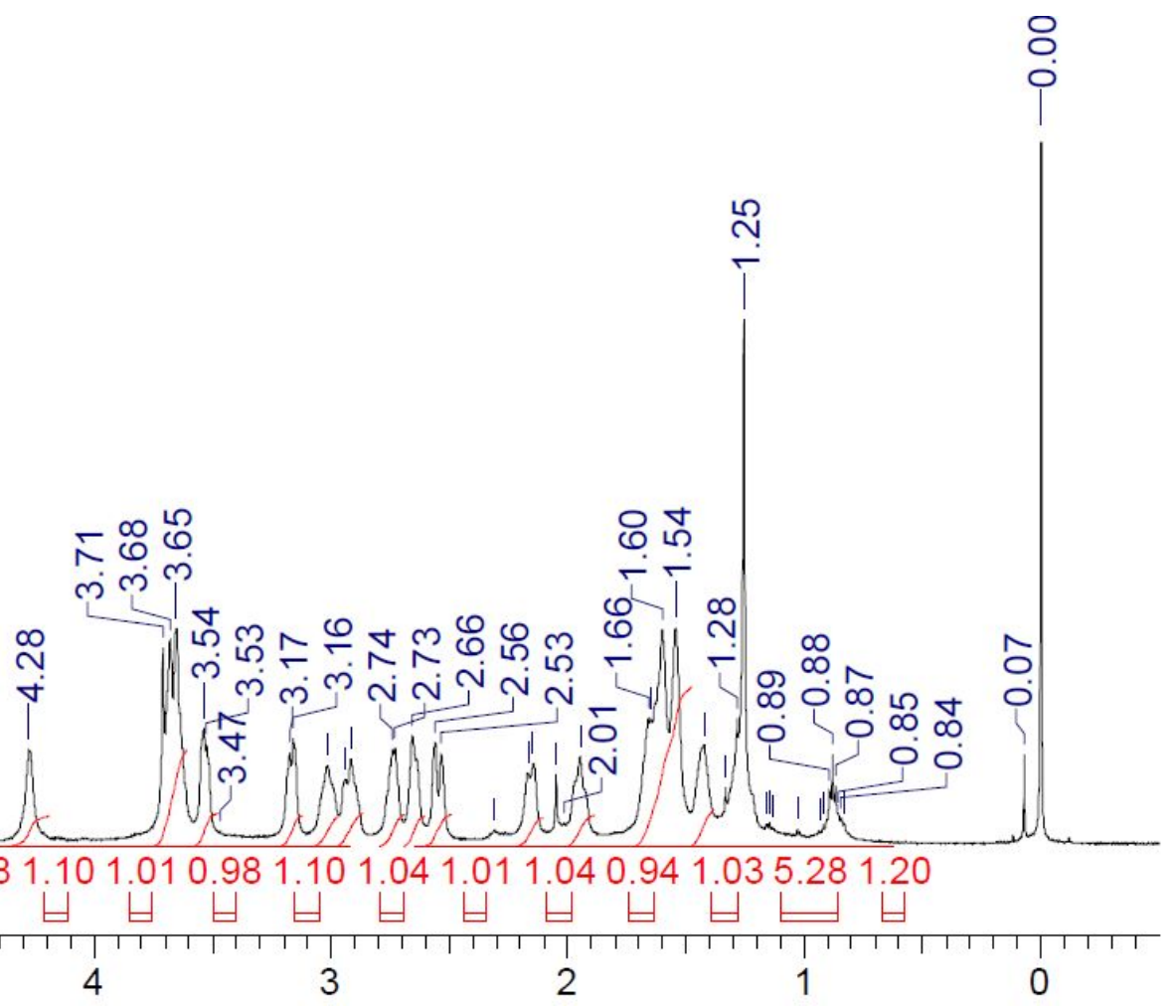


4-20. NMR Spectrum of 2-(1,2,3,4,6,7,12,12b-Octahydroindolo[2,3-a]quinolizin-2-yl)butane-1,4-diol (20-epi-28)

a) ${ }^{1} \mathrm{H}$ NMR Spectrum (in $\mathrm{CDCl}_{3}, 500 \mathrm{MHz}$ )

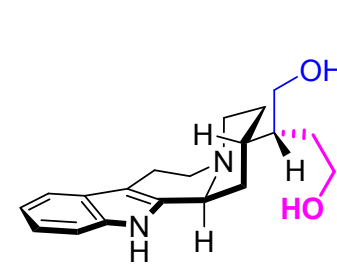

20-epi-28

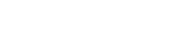

\section{,}

.
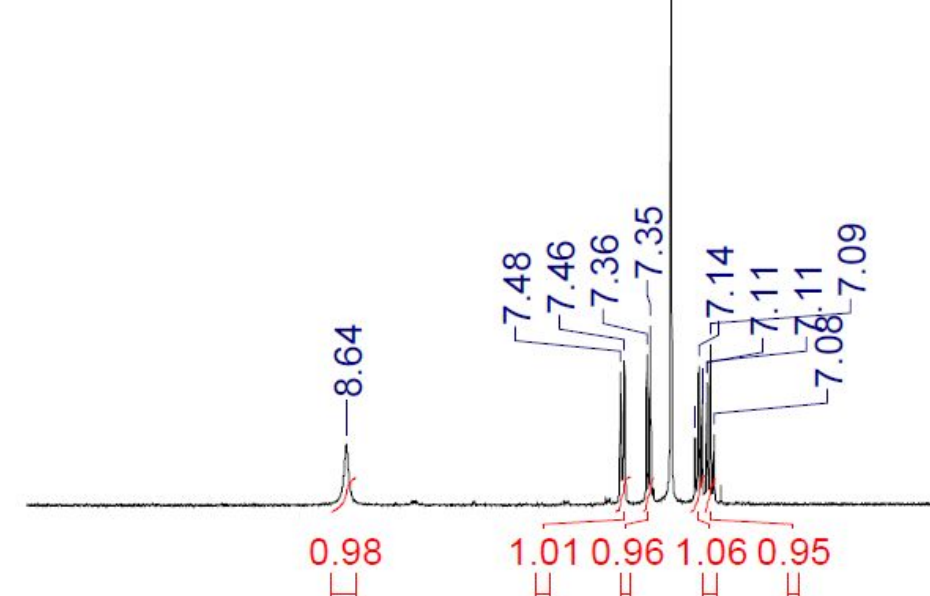

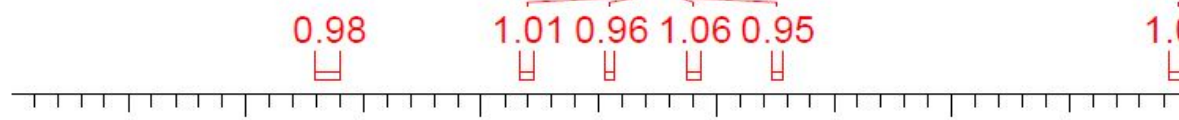

$\stackrel{\circ}{\mathrm{N}}$

7


$6 \quad 5$

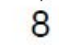

8
ఠ

$\checkmark \infty \infty$ $\forall \dot{L}$

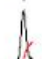

mon 00

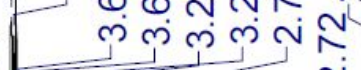
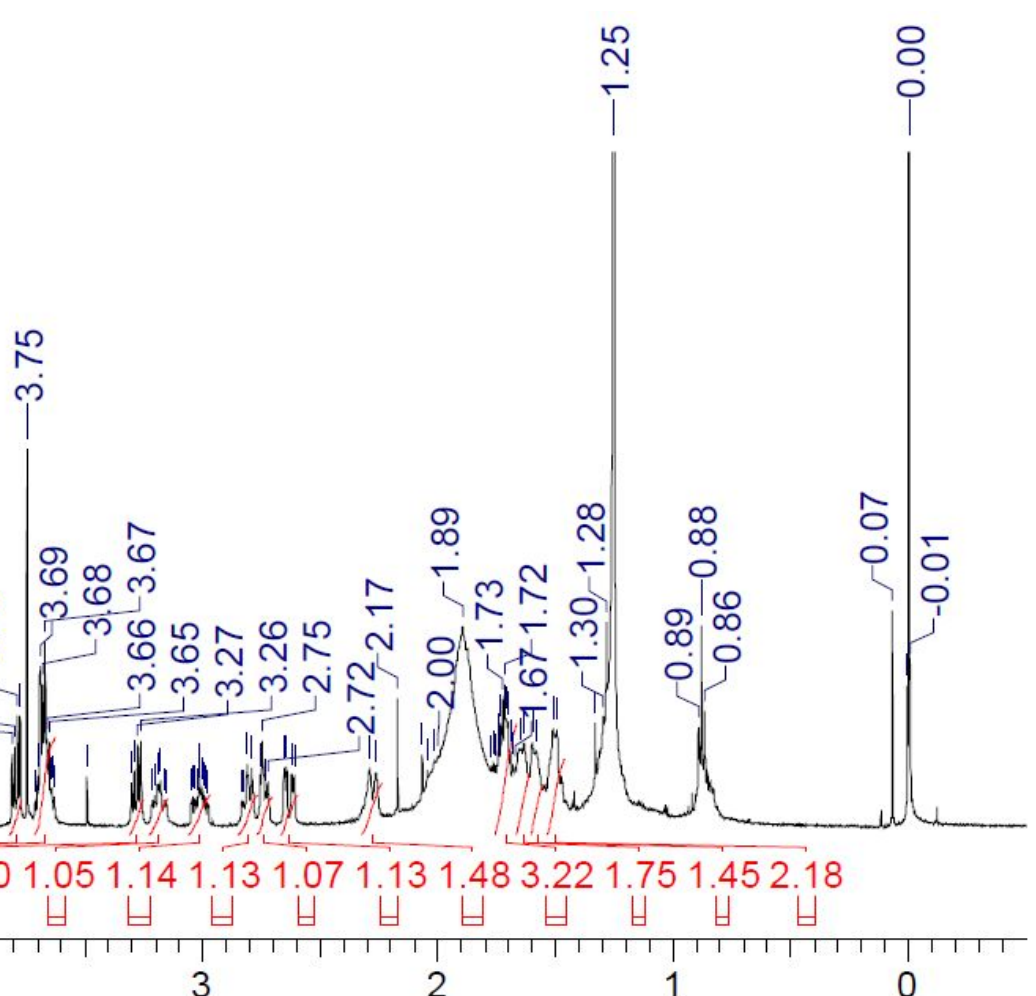
4-21. NMR Spectra of tert-Butyl 2-(1-Methoxy-1-oxobutan-2-yl)-1,3,4,6,7,12b-hexahydroindolo[2,3-a]quinolizine-12(2H)-carboxylate (29)

a) ${ }^{1} \mathrm{H}$ NMR Spectrum (in $\mathrm{CDCl}_{3}, 500 \mathrm{MHz}$ )
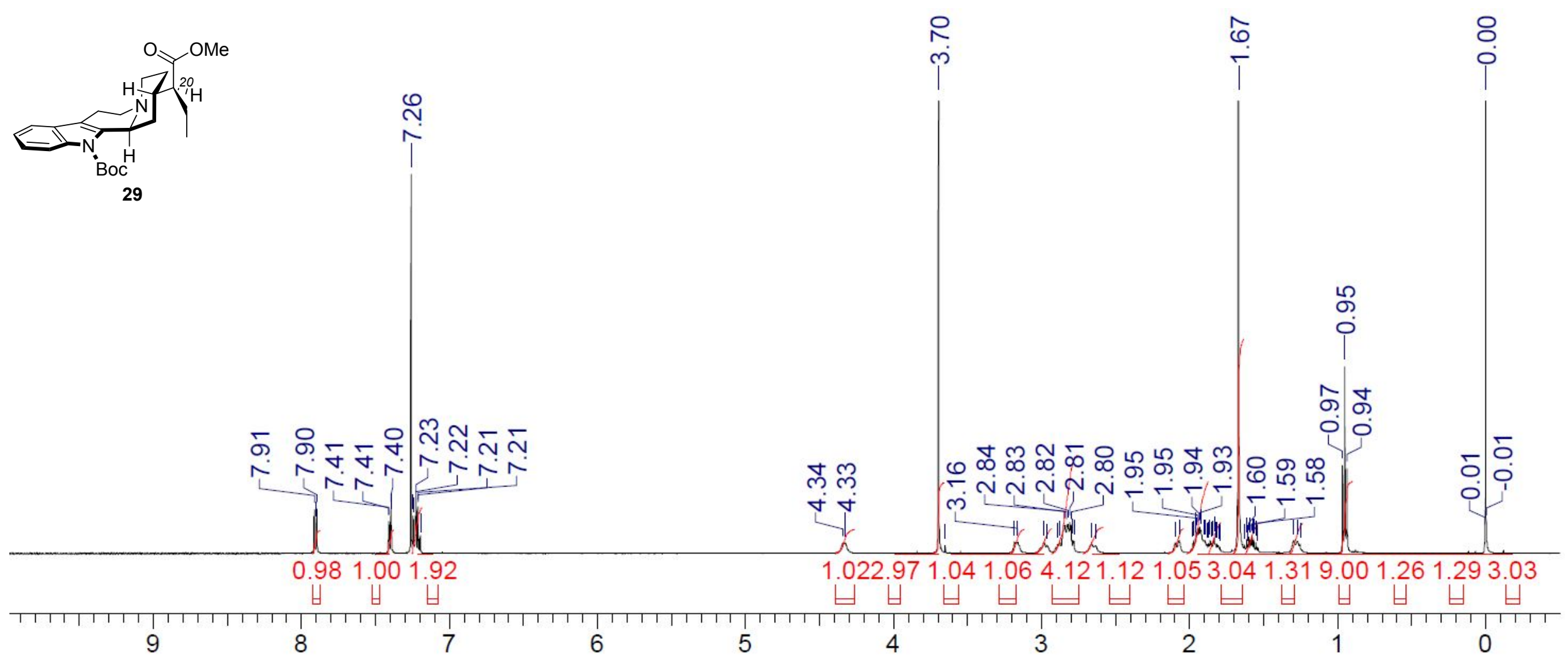
4-22. NMR Spectra of tert-Butyl 2-(1-Methoxy-1-oxobutan-2-yl)-1,3,4,6,7,12b-hexahydroindolo[2,3-a]quinolizine-12(2H)-carboxylate (20-epi-29)

a) ${ }^{1} \mathrm{H}$ NMR Spectrum (in $\mathrm{CDCl}_{3}, 500 \mathrm{MHz}$ )
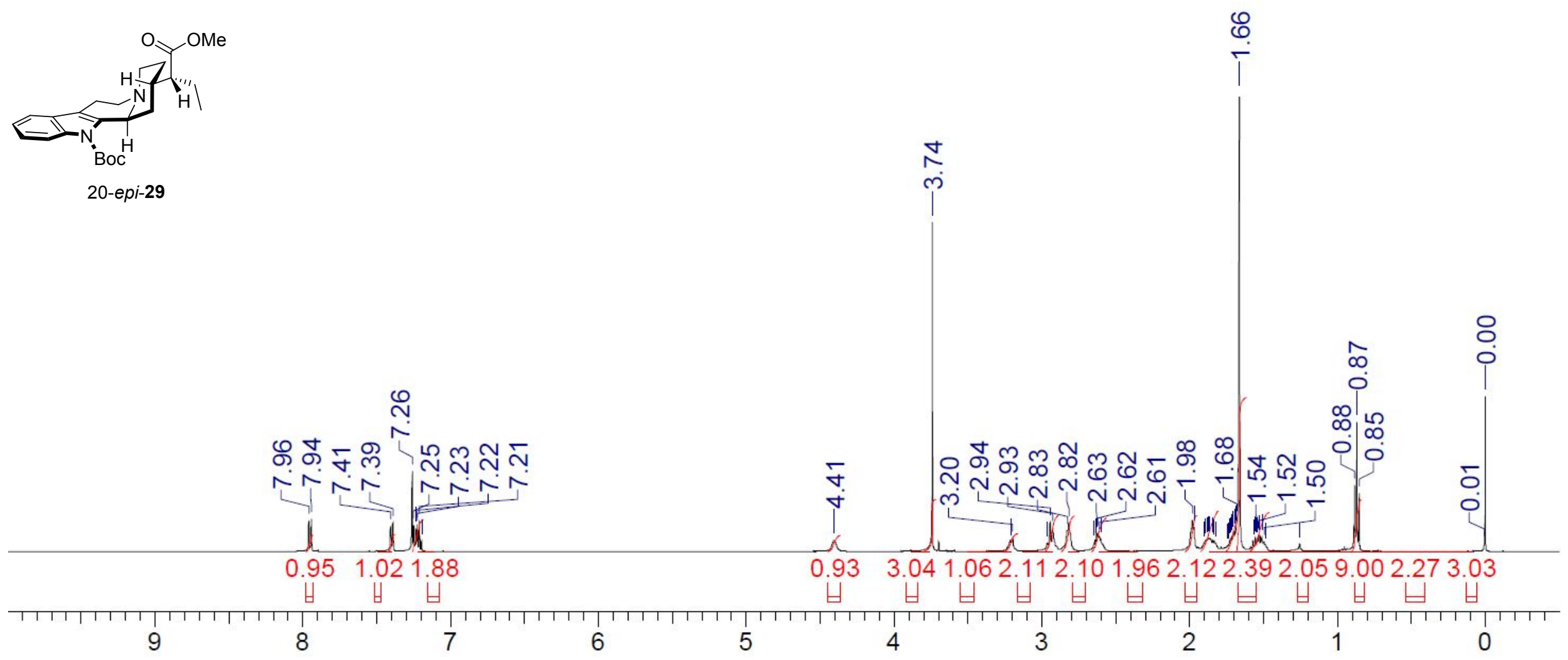
4-23. NMR Spectra of 18,19-Dihydroantirhine (1-H

a) ${ }^{1} \mathrm{H}$ NMR Spectrum (in $\mathrm{CDCl}_{3}, 500 \mathrm{MHz}$ )

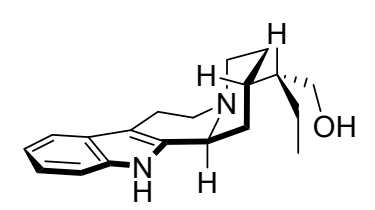

18,19-dihydroantirhine $\left(\mathbf{1}-\mathbf{H}_{\mathbf{2}}\right)$

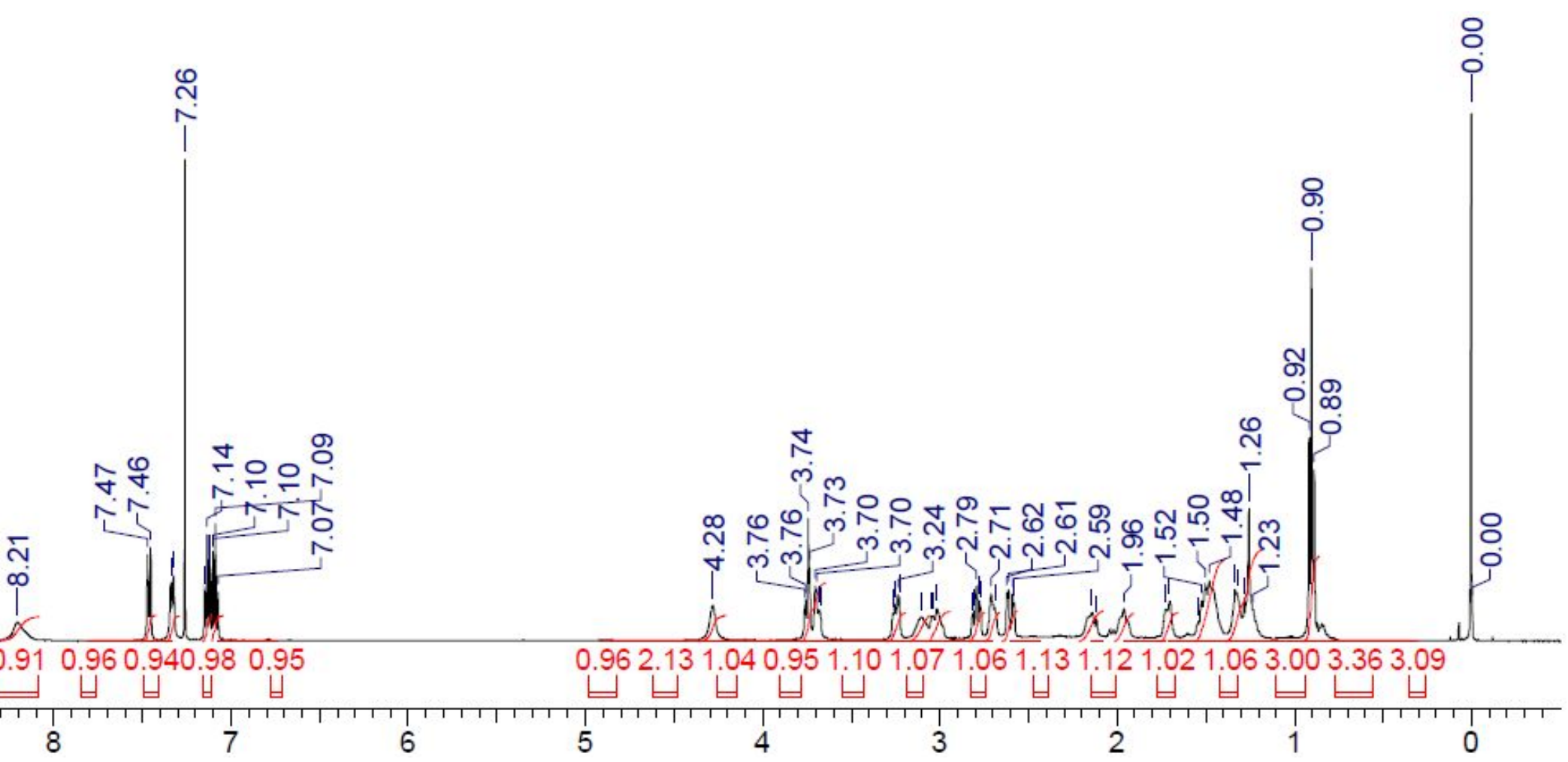


b) ${ }^{13} \mathrm{C}\left\{{ }^{1} \mathrm{H}\right\}$ NMR Spectrum (in $\mathrm{CDCl}_{3}, 125 \mathrm{MHz}$ )

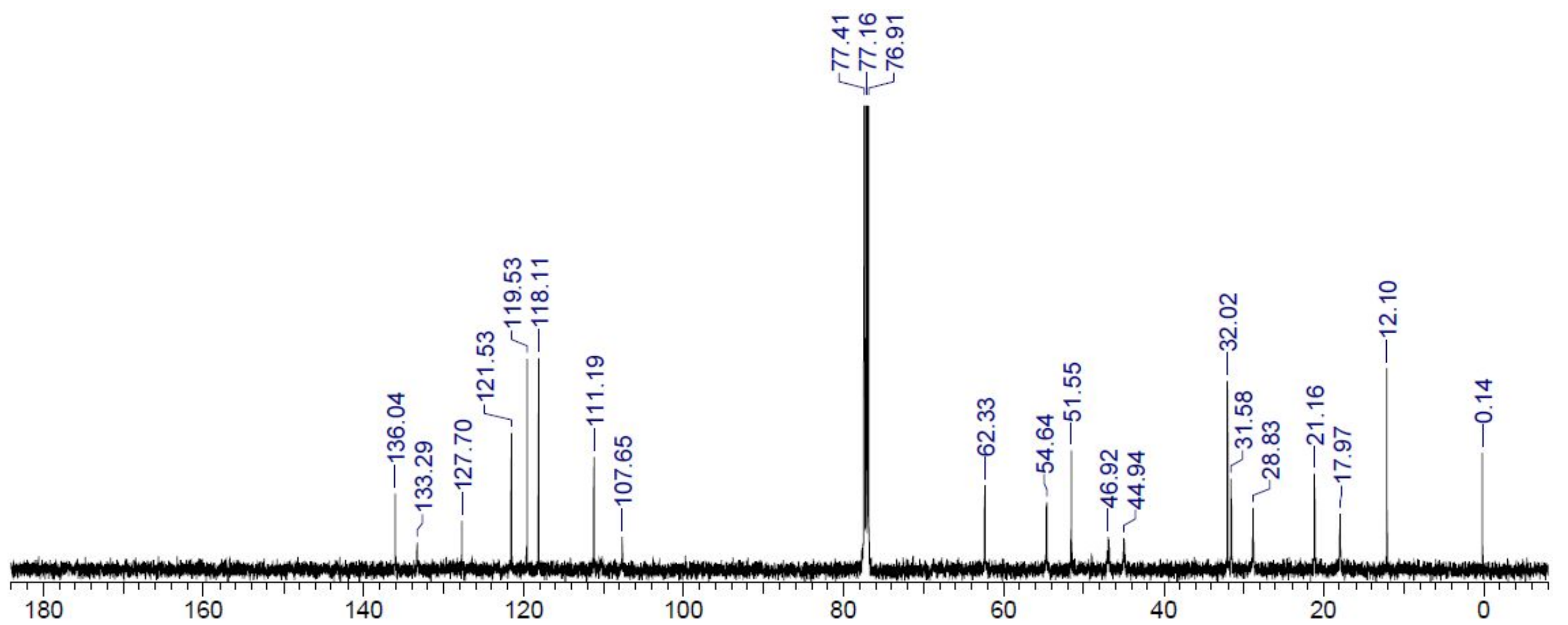


4-24. NMR Spectra of tert-Butyl 2-(1,3-Mimethoxy-1-oxopropan-2-yl)-1,3,4,6,7,12b-hexahydroindolo[2,3-a]quinolizine-12(2H)-carboxylate (31a)

a) ${ }^{1} \mathrm{H}$ NMR Spectrum (in $\mathrm{CDCl}_{3}, 500 \mathrm{MHz}$ )

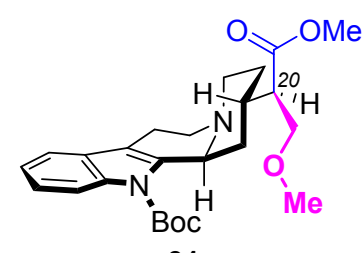

$31 a$

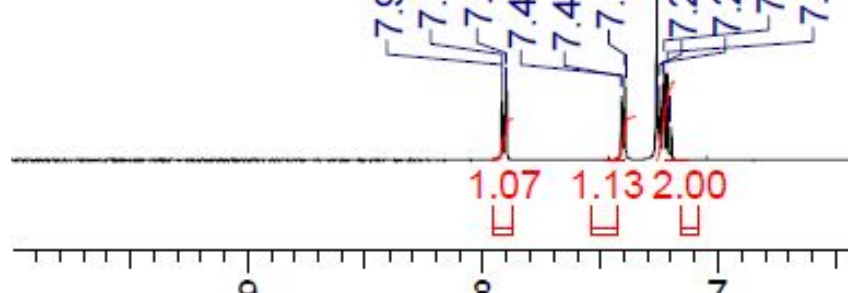

9

8

7

1.011 .033 .001 .063 .001 .111 .091 .093 .14

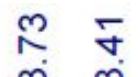

\&

8

\%

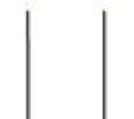

पूळ

mூm

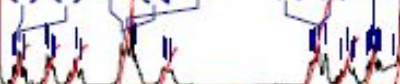

W.

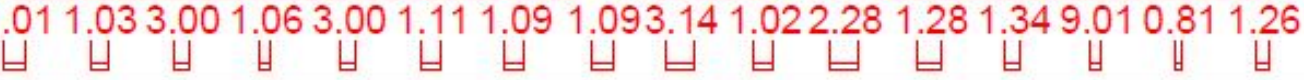

4

3

2

1 
b) ${ }^{13} \mathrm{C}\left\{{ }^{1} \mathrm{H}\right\}$ NMR Spectrum (in $\mathrm{CDCl}_{3}, 125 \mathrm{MHz}$ )

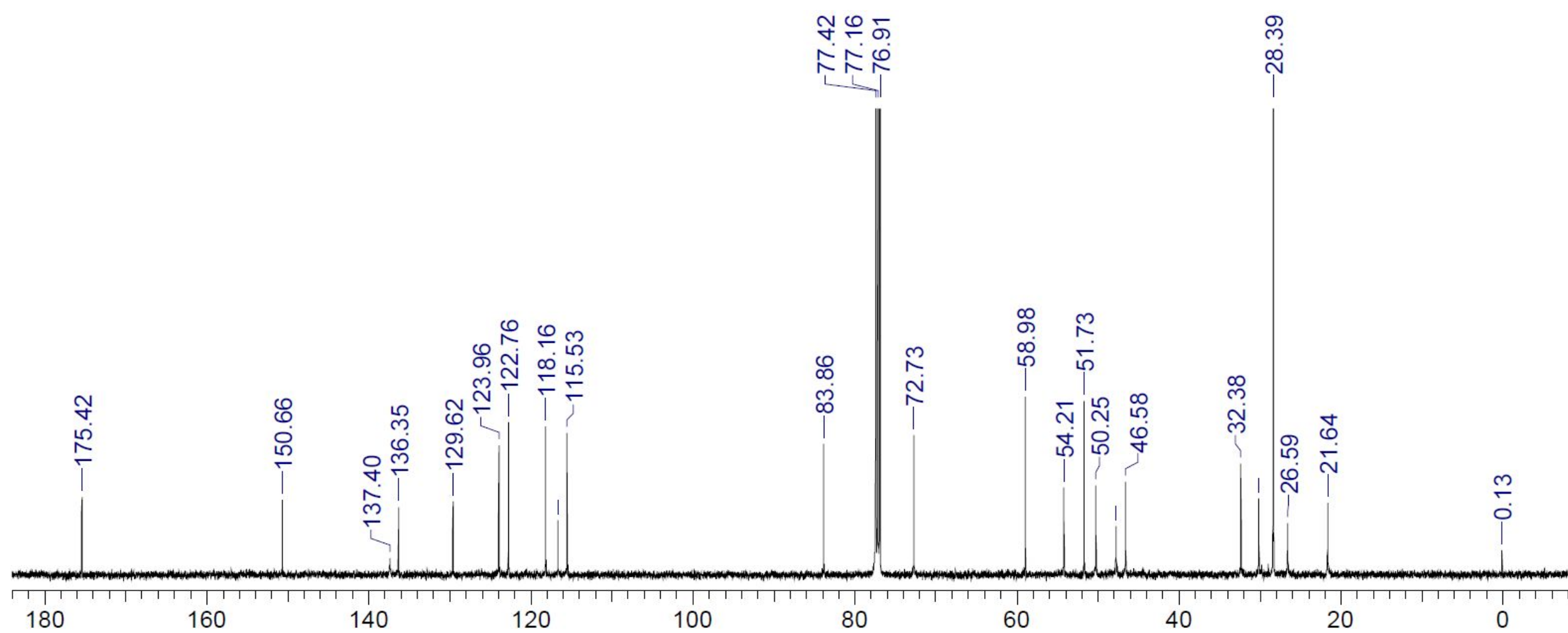


4-25. NMR Spectra of tert-Butyl 2-(1,3-Dimethoxy-1-oxopropan-2-yl)-1,3,4,6,7,12b-hexahydroindolo[2,3-a]quinolizine-12(2H)-carboxylate (20-epi-31a)

a) ${ }^{1} \mathrm{H}$ NMR Spectrum (in $\mathrm{CDCl}_{3}, 500 \mathrm{MHz}$ )

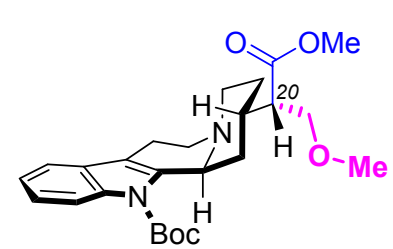

20-epi-31a

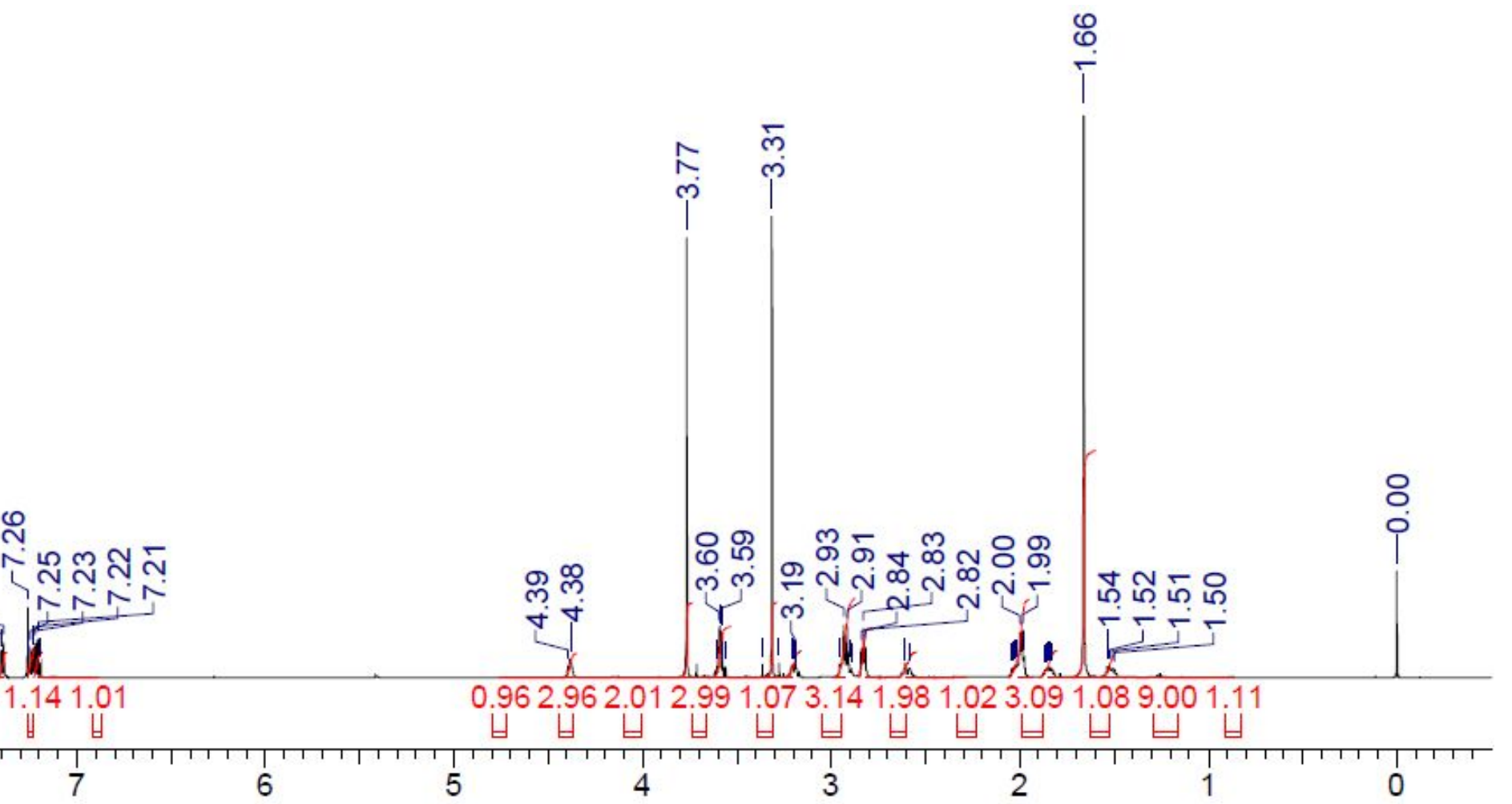


b) ${ }^{13} \mathrm{C}\left\{{ }^{1} \mathrm{H}\right\}$ NMR Spectrum (in $\mathrm{CDCl}_{3}, 125 \mathrm{MHz}$ )

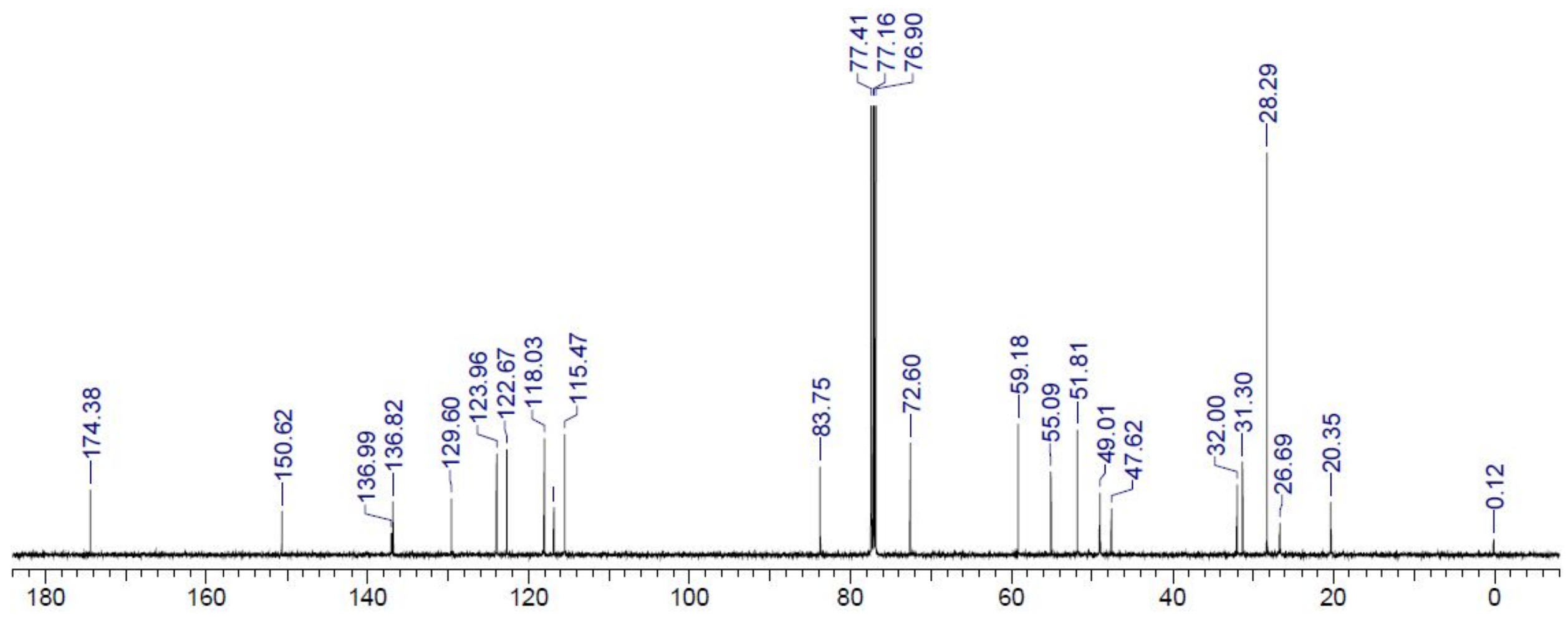


4-26. NMR Spectra of tert-Butyl 2-(3-(Benzyloxy)-1-methoxy-1-oxopropan-2-yl)-1,3,4,6,7,12b-hexahydroindolo[2,3-a]quinolizine-12(2H)-carboxylate (31b)

a) ${ }^{1} \mathrm{H}$ NMR Spectrum (in $\mathrm{CDCl}_{3}, 500 \mathrm{MHz}$ )
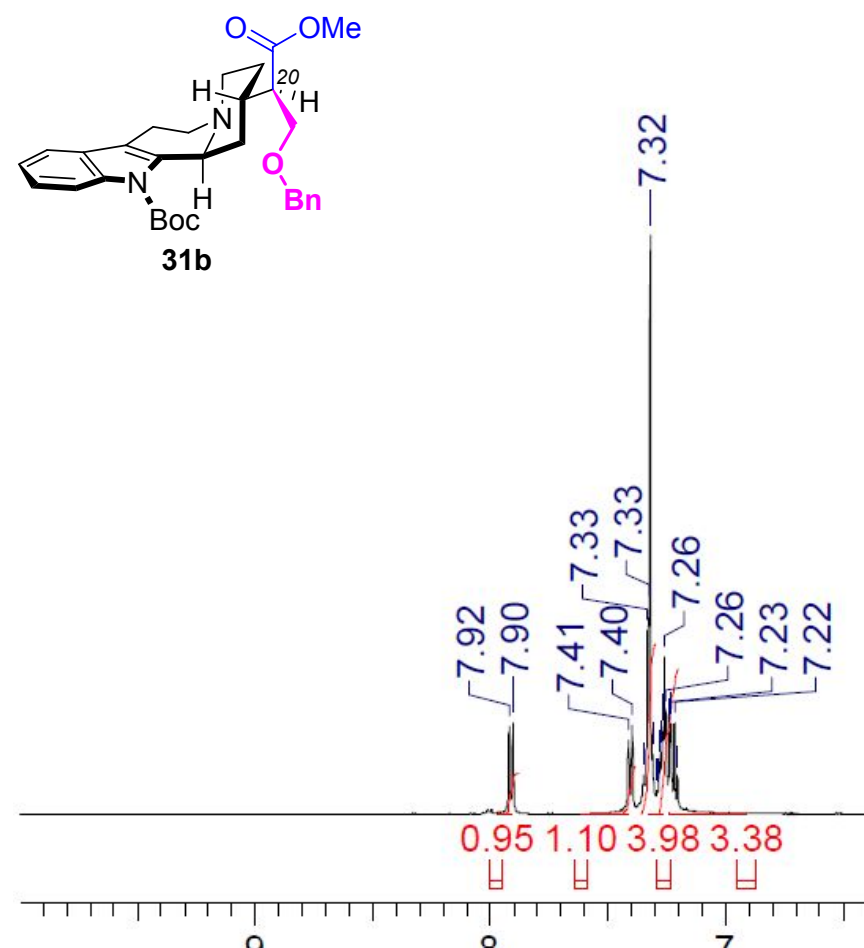

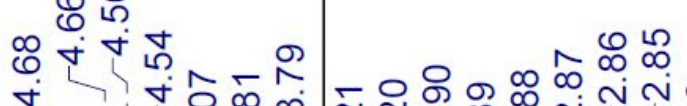

e

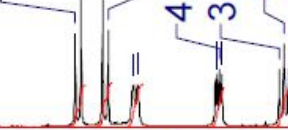

$\forall$

4

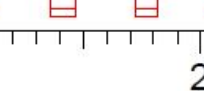

2

1

0 
b) ${ }^{13} \mathrm{C}\left\{{ }^{1} \mathrm{H}\right\}$ NMR Spectrum (in $\mathrm{CDCl}_{3}, 125 \mathrm{MHz}$ )

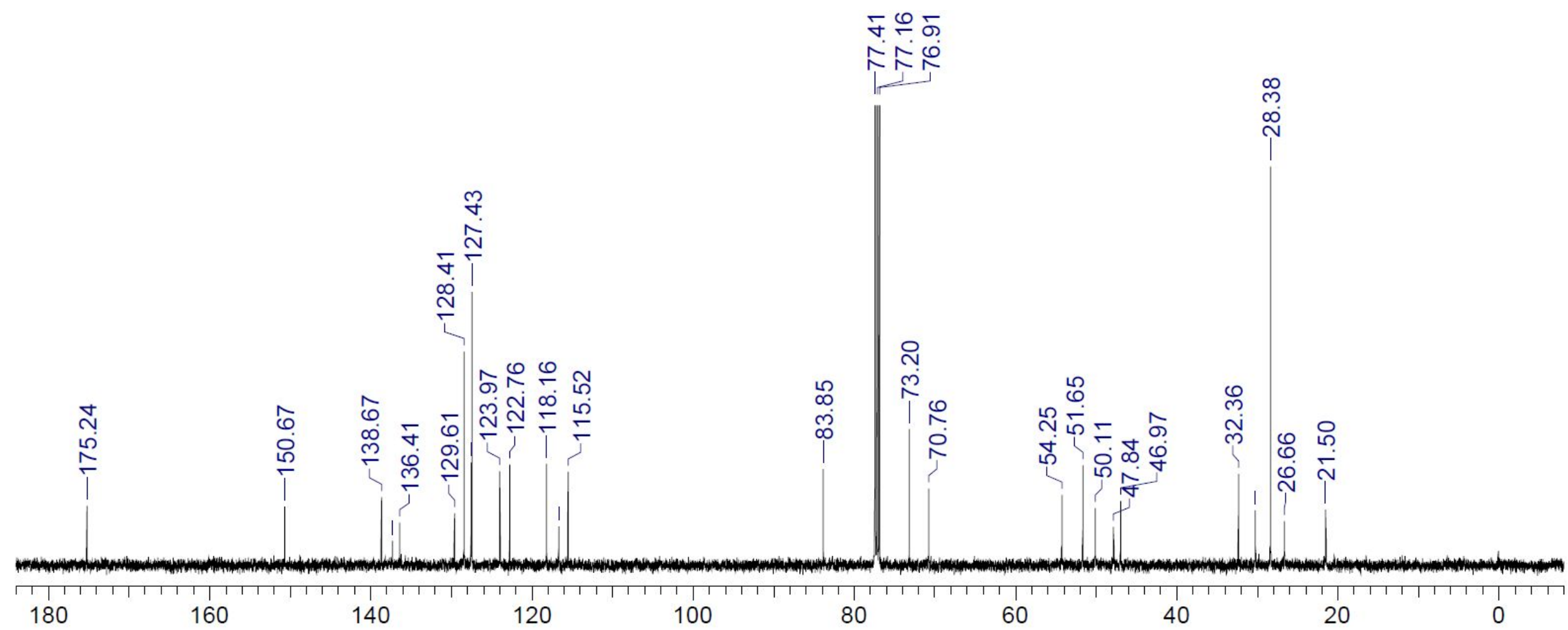


4-27. NMR Spectra of tert-Butyl 2-(3-(Benzyloxy)-1-methoxy-1-oxopropan-2-yl)-1,3,4,6,7,12b-hexahydroindolo[2,3-a]quinolizine-12(2H)-carboxylate (20-epi-31b) a) ${ }^{1} \mathrm{H}$ NMR Spectrum (in $\mathrm{CDCl}_{3}, 500 \mathrm{MHz}$ )

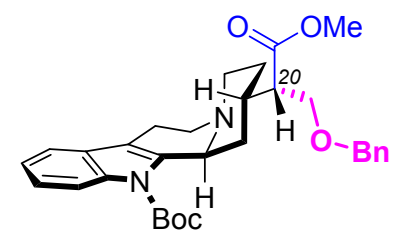

20-epi-31b

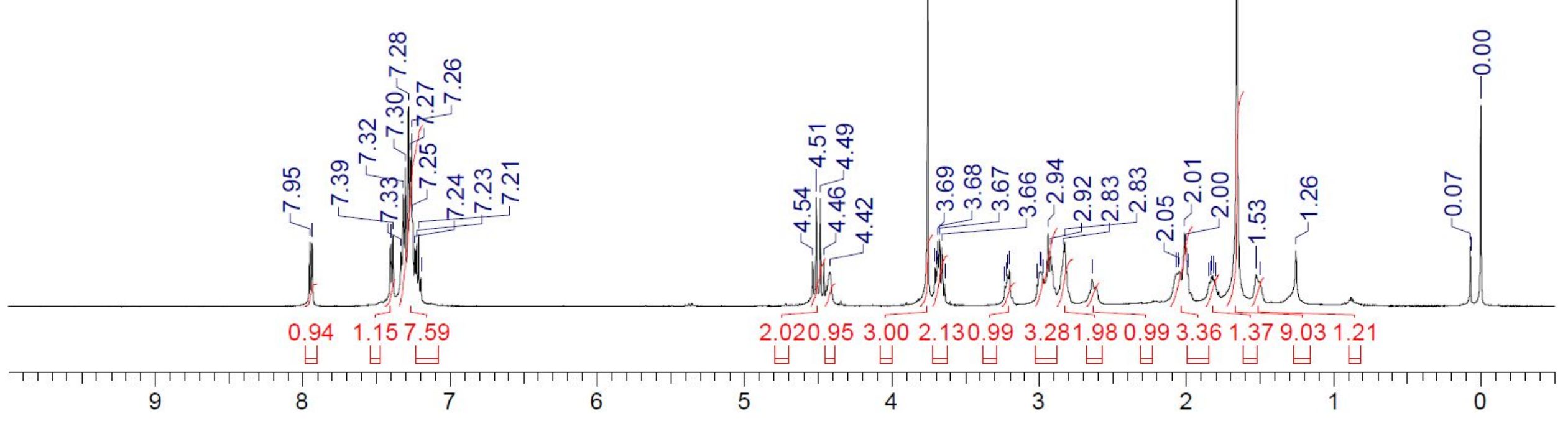


b) ${ }^{13} \mathrm{C}\left\{{ }^{1} \mathrm{H}\right\}$ NMR Spectrum (in $\mathrm{CDCl}_{3}, 125 \mathrm{MHz}$ )

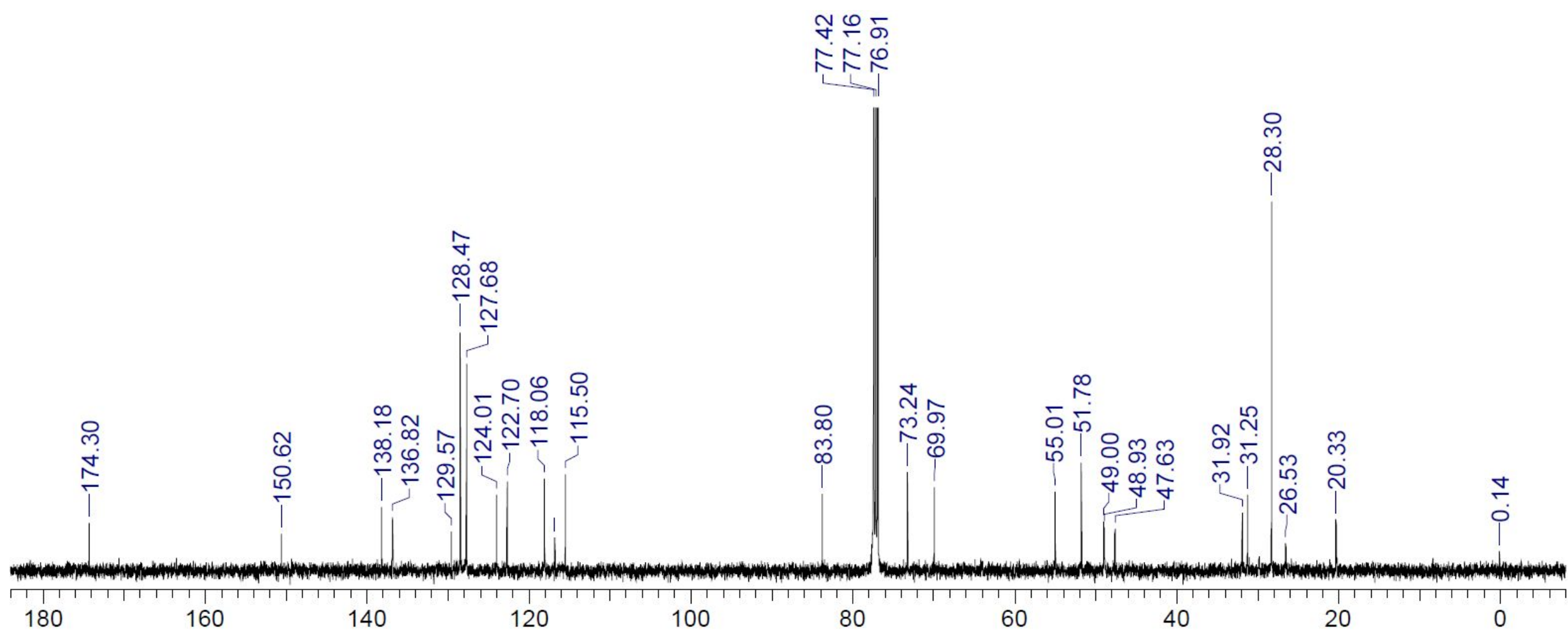




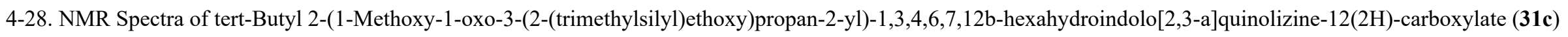
a) ${ }^{1} \mathrm{H}$ NMR Spectrum (in $\mathrm{CDCl}_{3}, 500 \mathrm{MHz}$ )
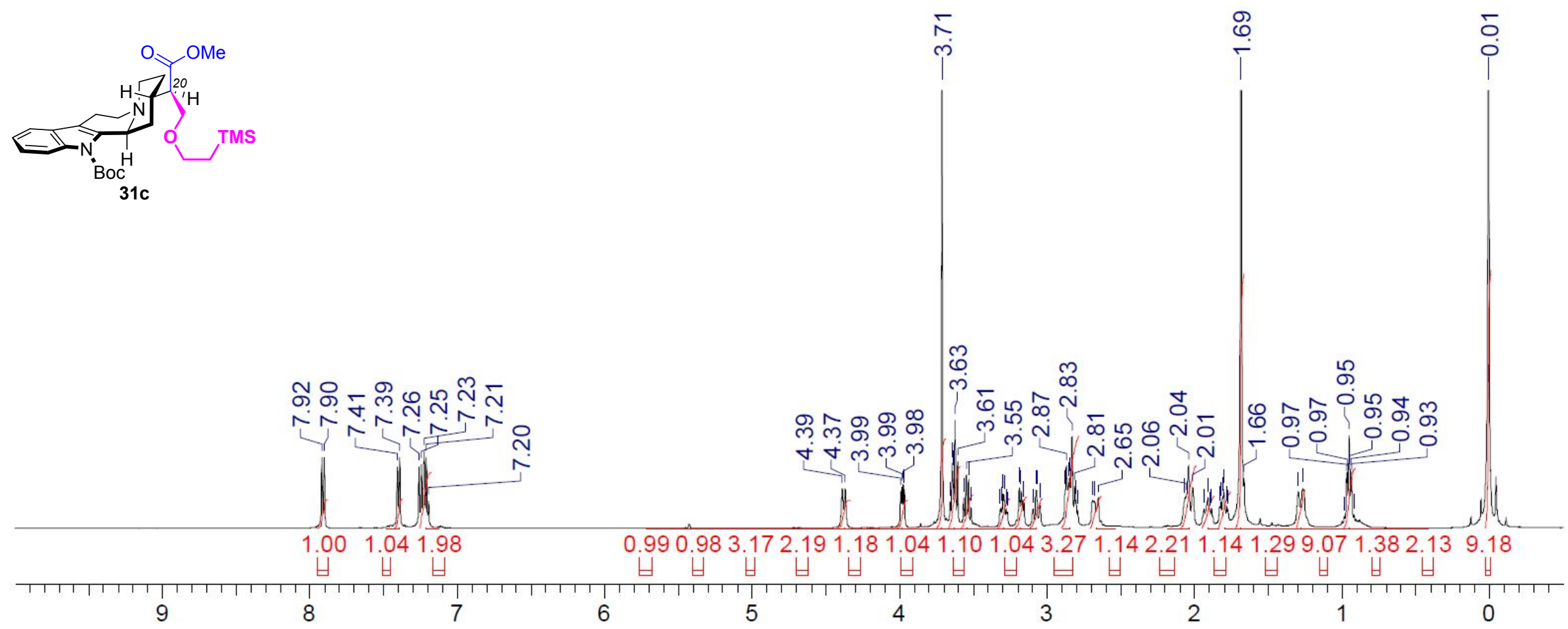
b) ${ }^{13} \mathrm{C}\left\{{ }^{1} \mathrm{H}\right\}$ NMR Spectrum (in $\mathrm{CDCl}_{3}, 125 \mathrm{MHz}$ )

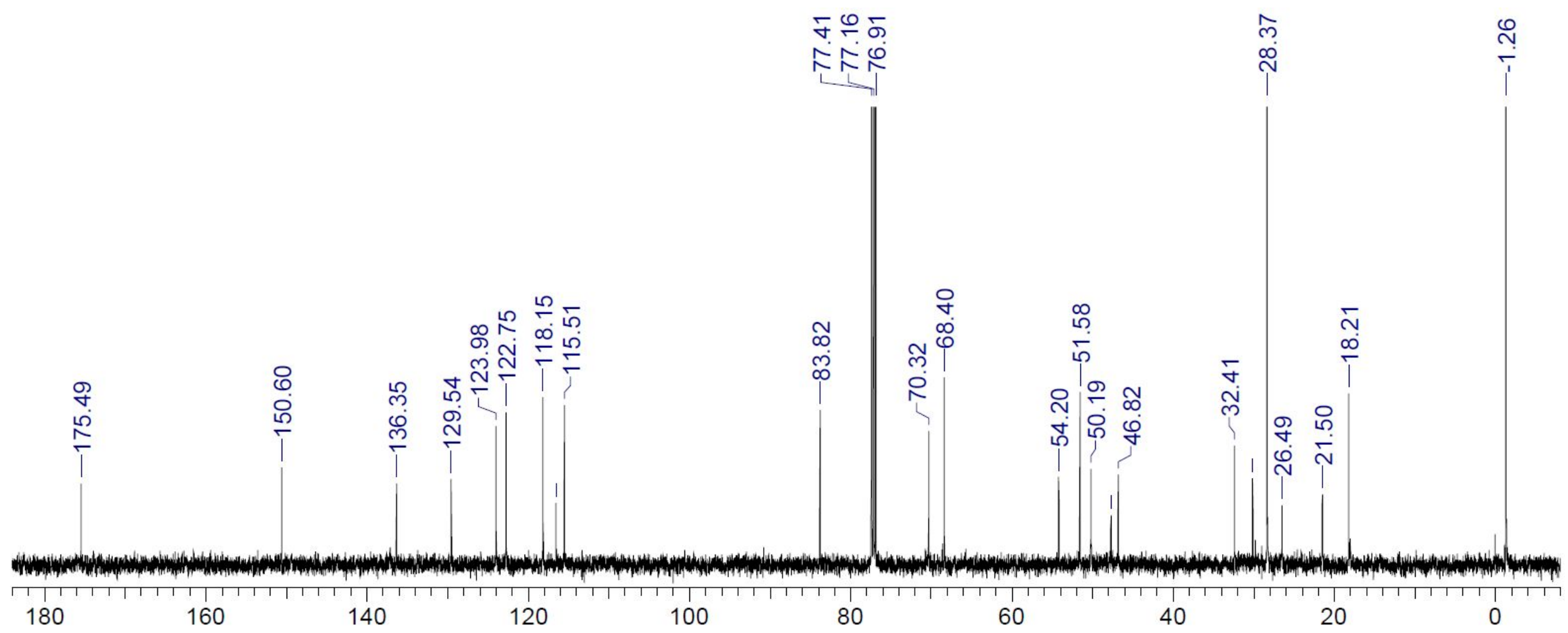


4-29. NMR Spectra of tert-Butyl 2-(1-Methoxy-1-oxo-3-(2-(trimethylsilyl)ethoxy)propan-2-yl)-1,3,4,6,7,12b-hexahydroindolo[2,3-a]quinolizine-12(2H)-carboxylate (20-epi-31c) a) ${ }^{1} \mathrm{H}$ NMR Spectrum (in $\mathrm{CDCl}_{3}, 500 \mathrm{MHz}$ )
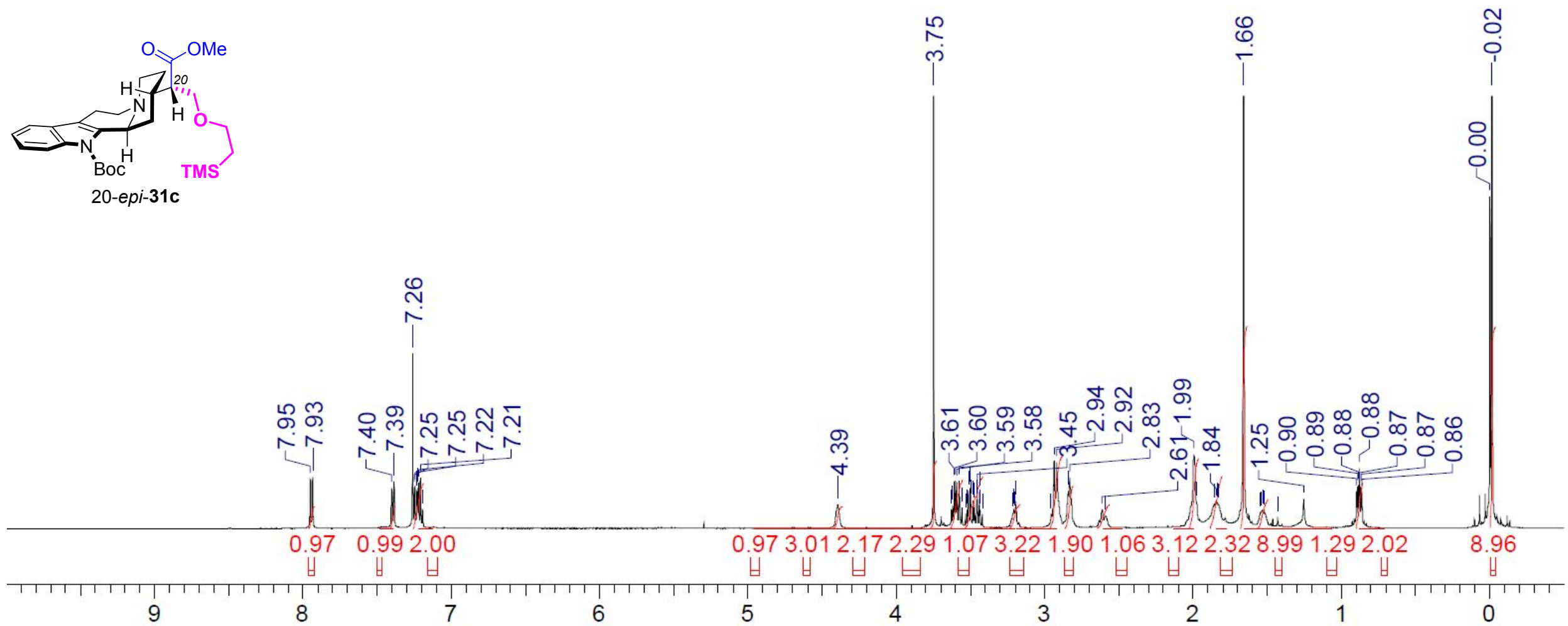
b) ${ }^{13} \mathrm{C}\left\{{ }^{1} \mathrm{H}\right\}$ NMR Spectrum (in $\mathrm{CDCl}_{3}, 125 \mathrm{MHz}$ )

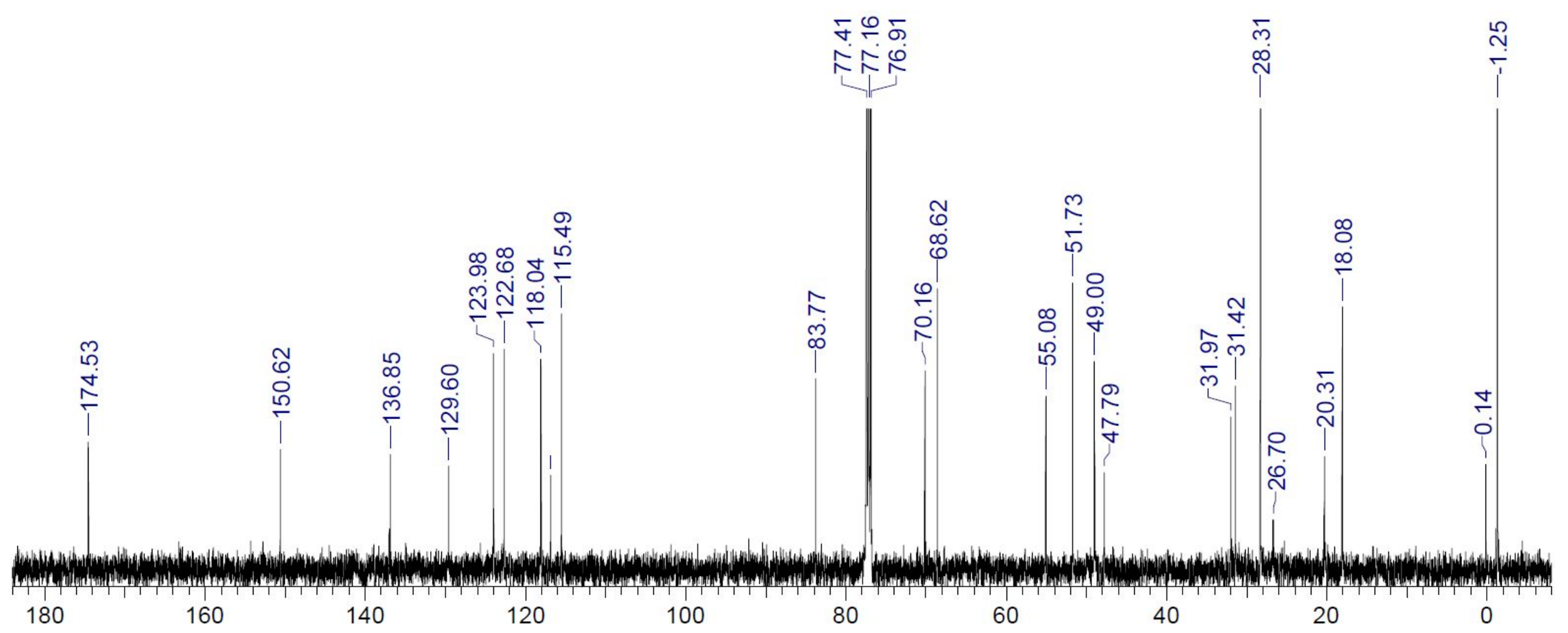


4-30. NMR Spectra of tert-Butyl 2-(1-Methoxy-3-(2-methoxyethoxy)-1-oxopropan-2-yl)-1,3,4,6,7,12b-hexahydroindolo[2,3-a]quinolizine-12(2H)-carboxylate (31d) a) ${ }^{1} \mathrm{H}$ NMR Spectrum (in $\mathrm{CDCl}_{3}, 500 \mathrm{MHz}$ )
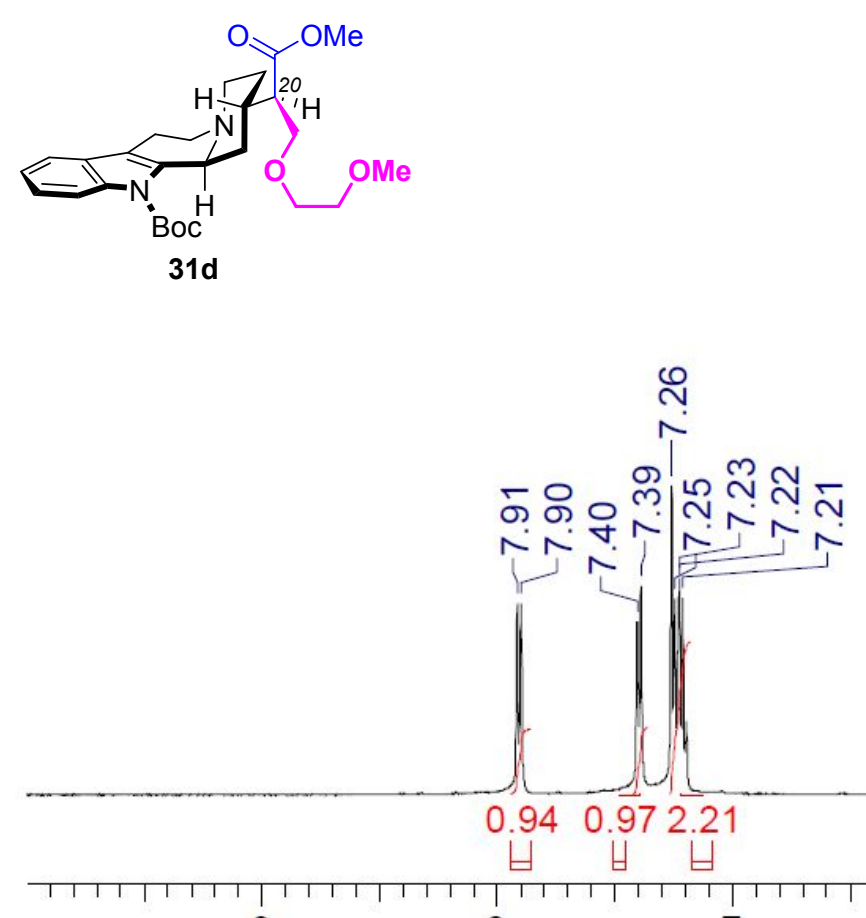

0.920 .985041282042871 .051 .040982901002 .421341 .239011 .27

$\begin{array}{llll}\bar{n} & \hat{m} & \infty & 0\end{array}$

(

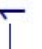

5

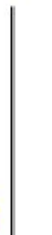

(1)

(1)

-

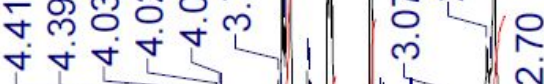

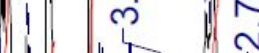
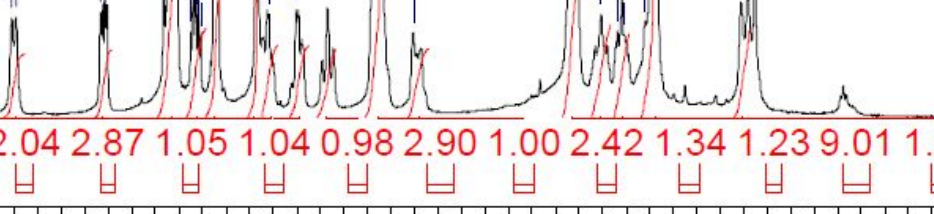

8

6

5

2 
b) ${ }^{13} \mathrm{C}\left\{{ }^{1} \mathrm{H}\right\}$ NMR Spectrum (in $\mathrm{CDCl}_{3}, 125 \mathrm{MHz}$ )

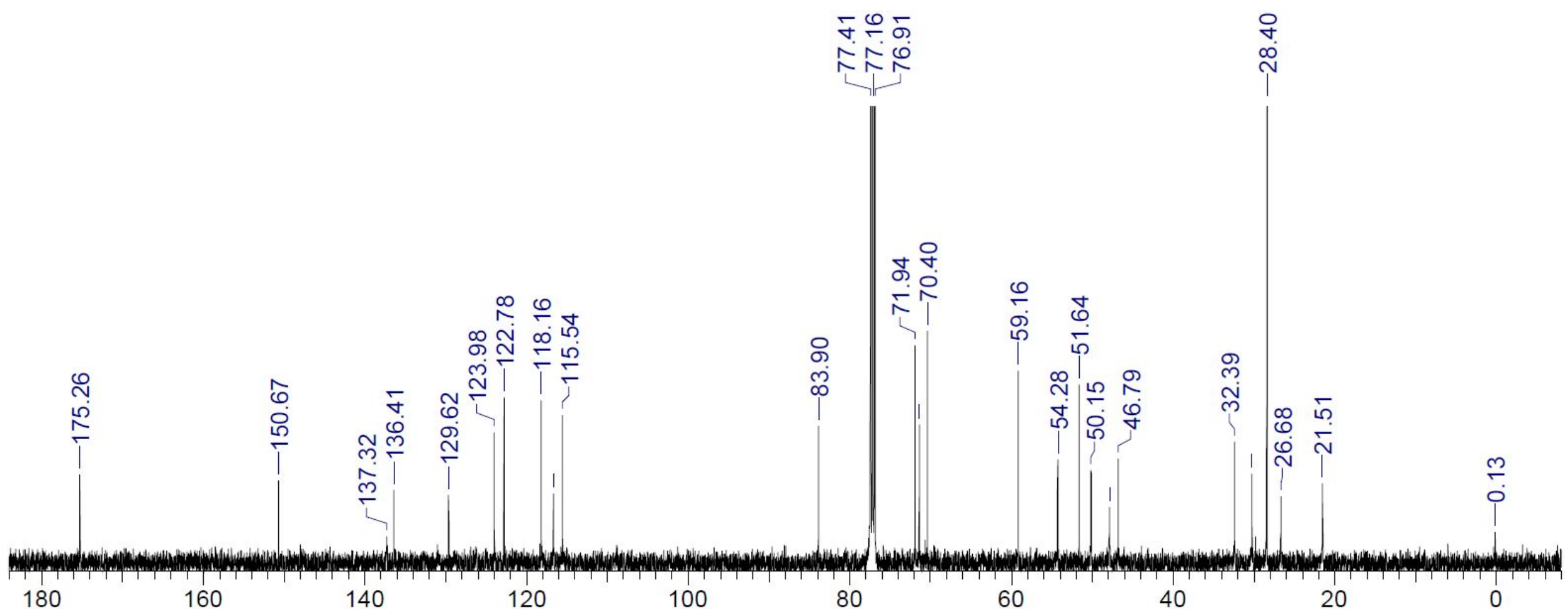


4-31. NMR Spectra of tert-Butyl 2-(1-Methoxy-3-(2-methoxyethoxy)-1-oxopropan-2-yl)-1,3,4,6,7,12b-hexahydroindolo[2,3-a]quinolizine-12(2H)-carboxylate (20-epi-31d) a) ${ }^{1} \mathrm{H}$ NMR Spectrum (in $\mathrm{CDCl}_{3}, 500 \mathrm{MHz}$ )

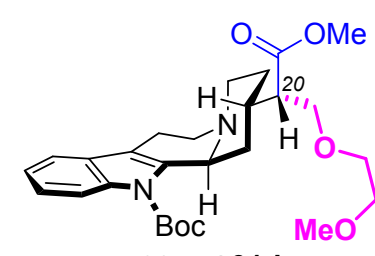

20-epi-31d $\stackrel{\leftrightarrow}{ヘ}$

N

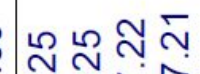

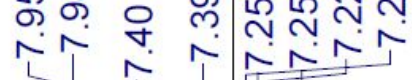

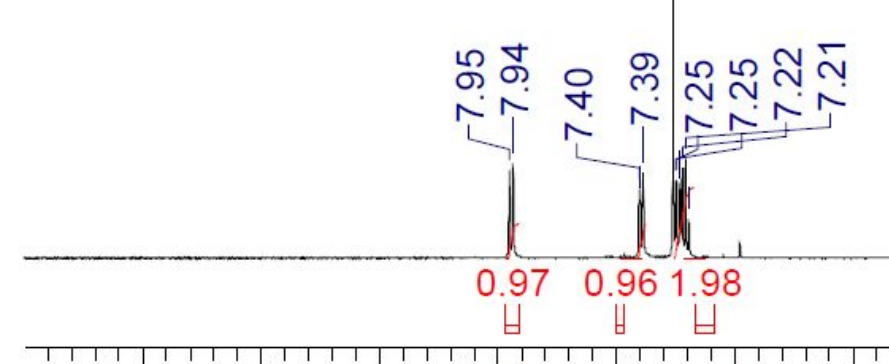

9
8

\begin{abstract}
7
\end{abstract}

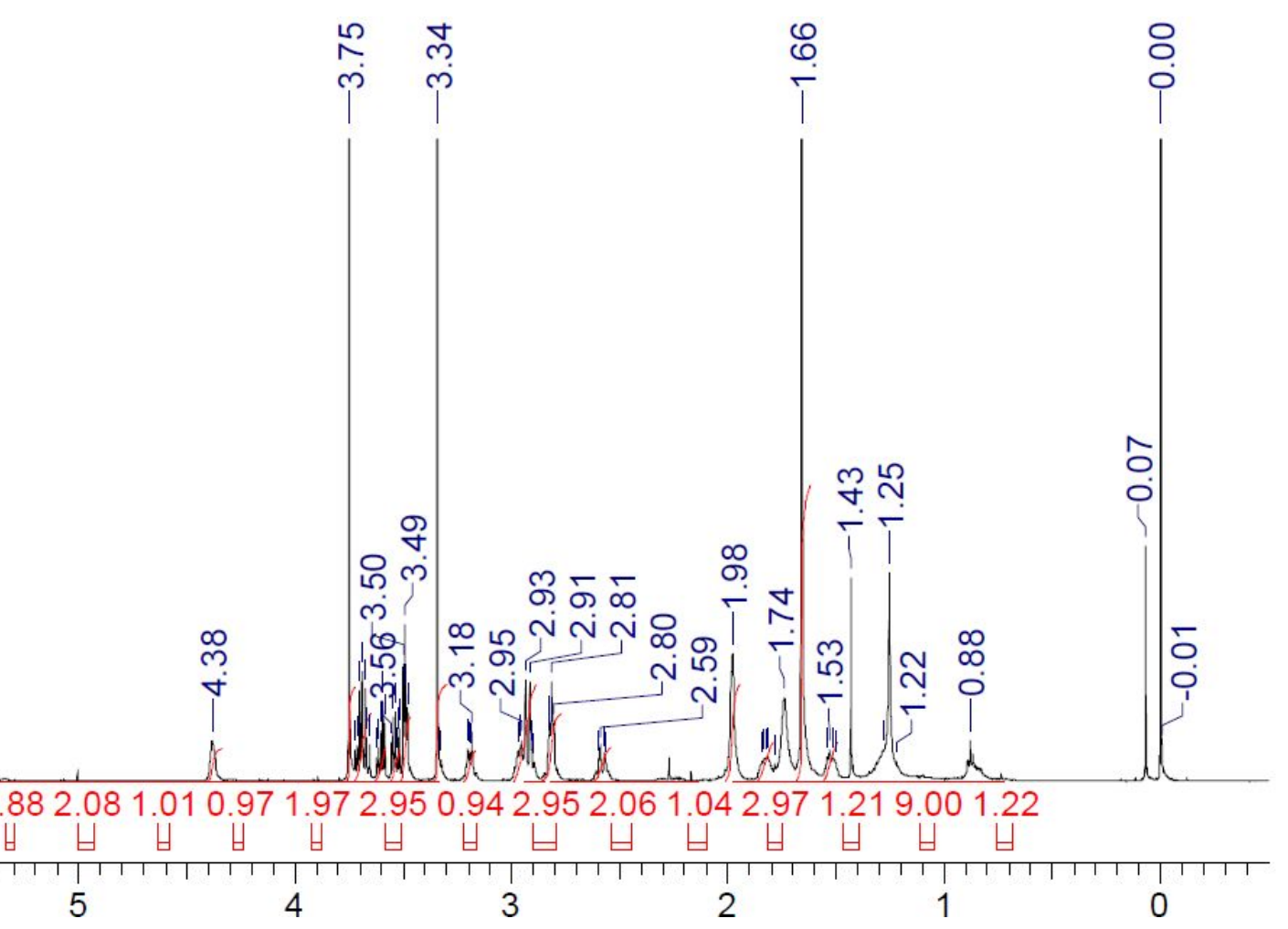


b) ${ }^{13} \mathrm{C}\left\{{ }^{1} \mathrm{H}\right\}$ NMR Spectrum (in $\mathrm{CDCl}_{3}, 125 \mathrm{MHz}$ )

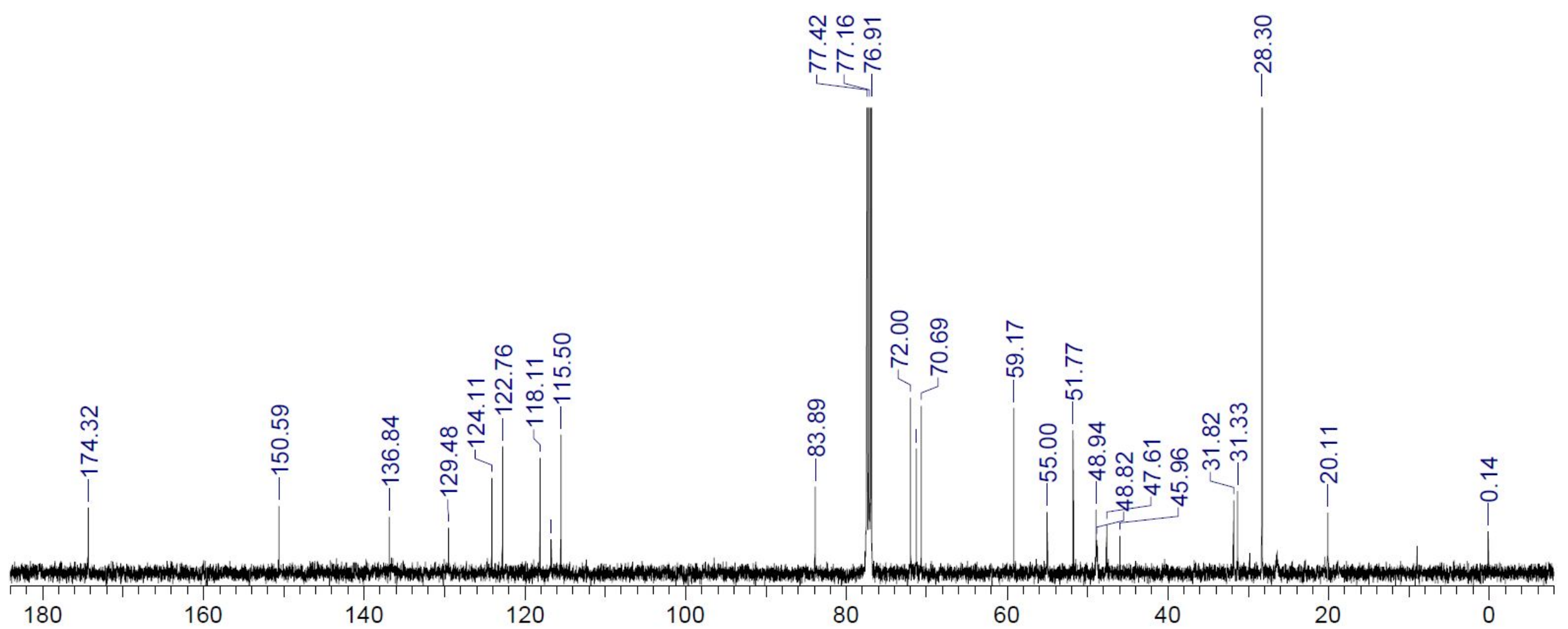


4-32. NMR Spectra of tert-Butyl 2-(1-Hydroxy-3-(2-(trimethylsilyl)ethoxy)propan-2-yl)-1,3,4,6,7,12b-hexahydroindolo[2,3-a]quinolizine-12(2H)-carboxylate (32)

a) ${ }^{1} \mathrm{H}$ NMR Spectrum (in $\mathrm{CDCl}_{3}, 500 \mathrm{MHz}$ )

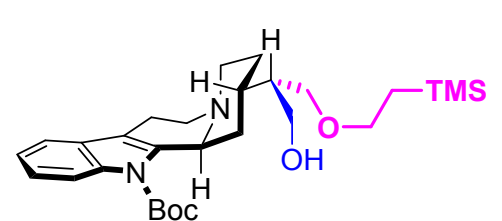

32

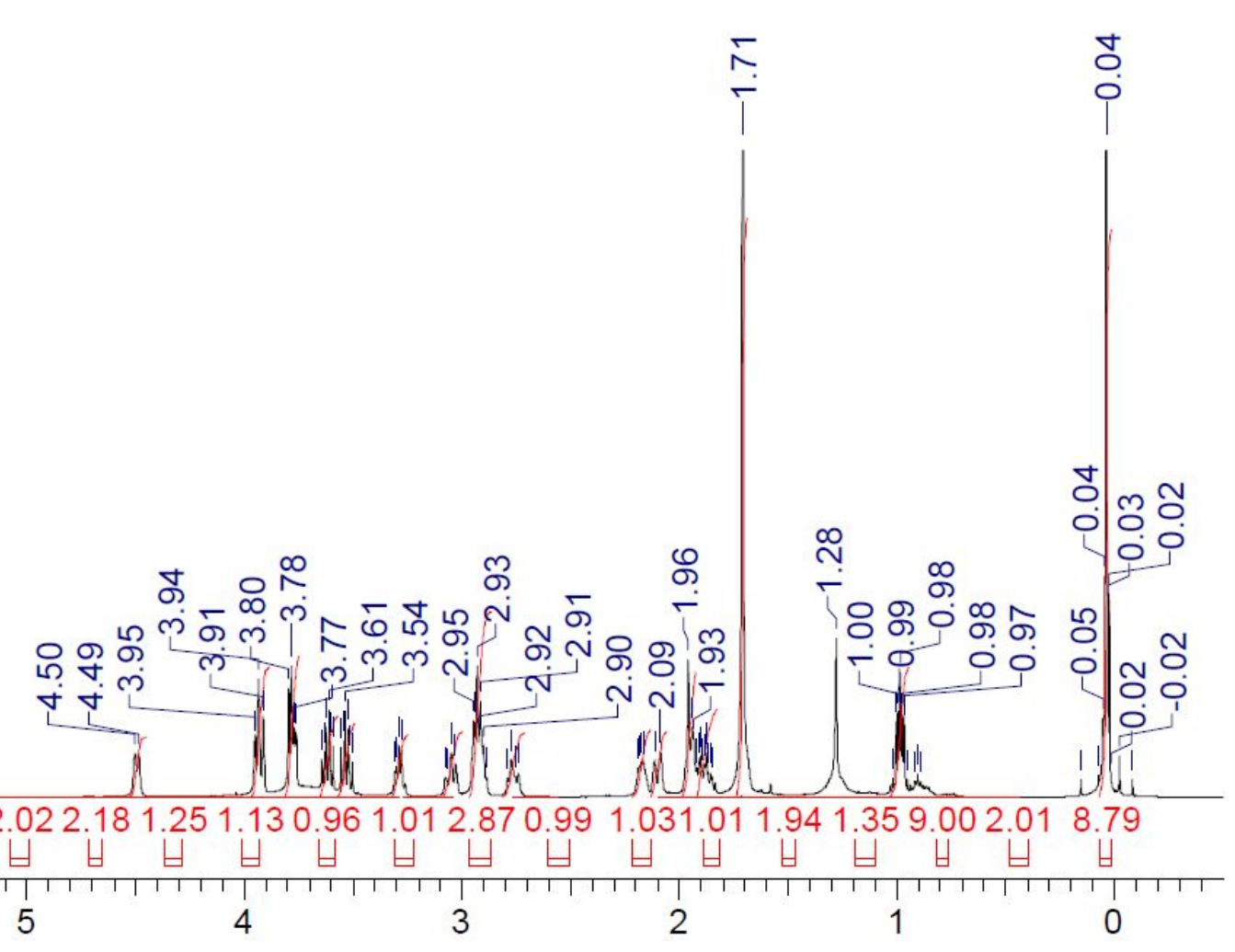


b) ${ }^{13} \mathrm{C}\left\{{ }^{1} \mathrm{H}\right\}$ NMR Spectrum (in $\mathrm{CDCl}_{3}, 125 \mathrm{MHz}$ )

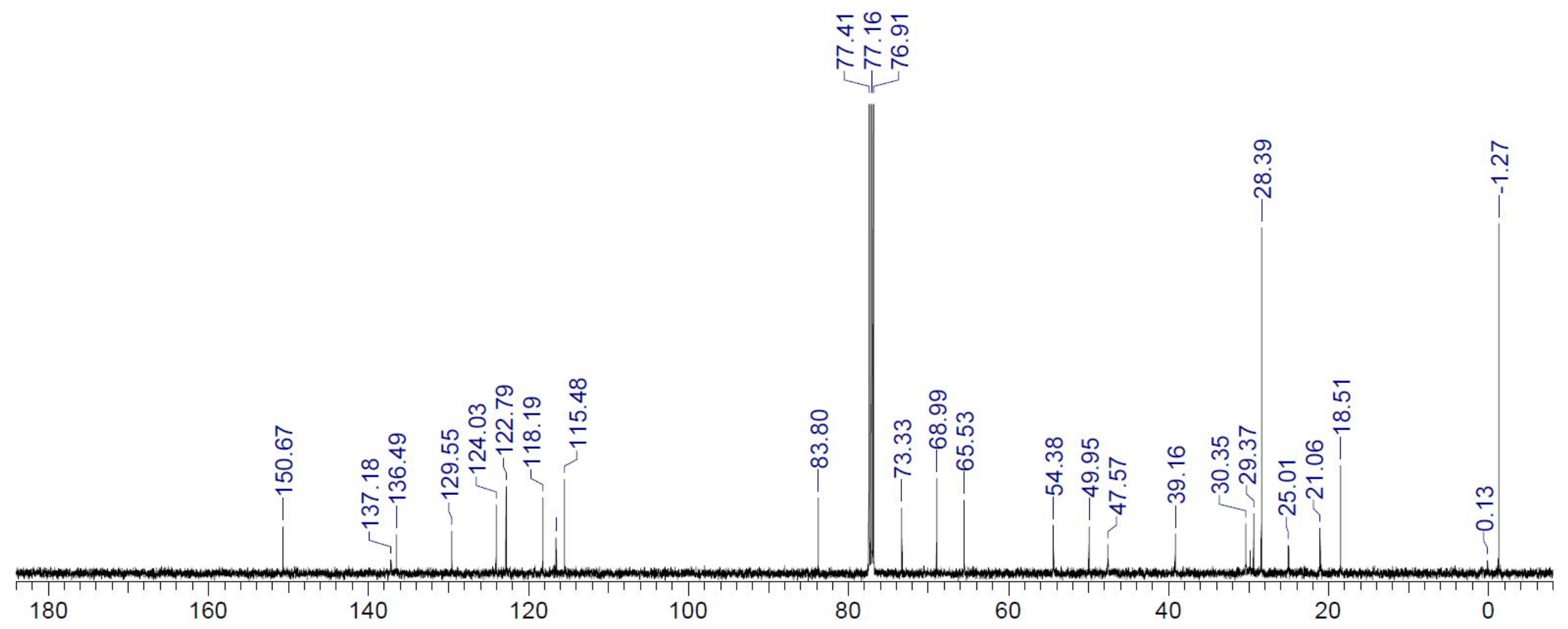


4-33. NMR Spectra of Antirhine (1)

a) ${ }^{1} \mathrm{H}$ NMR Spectrum (in $\mathrm{CDCl}_{3}, 500 \mathrm{MHz}$ )

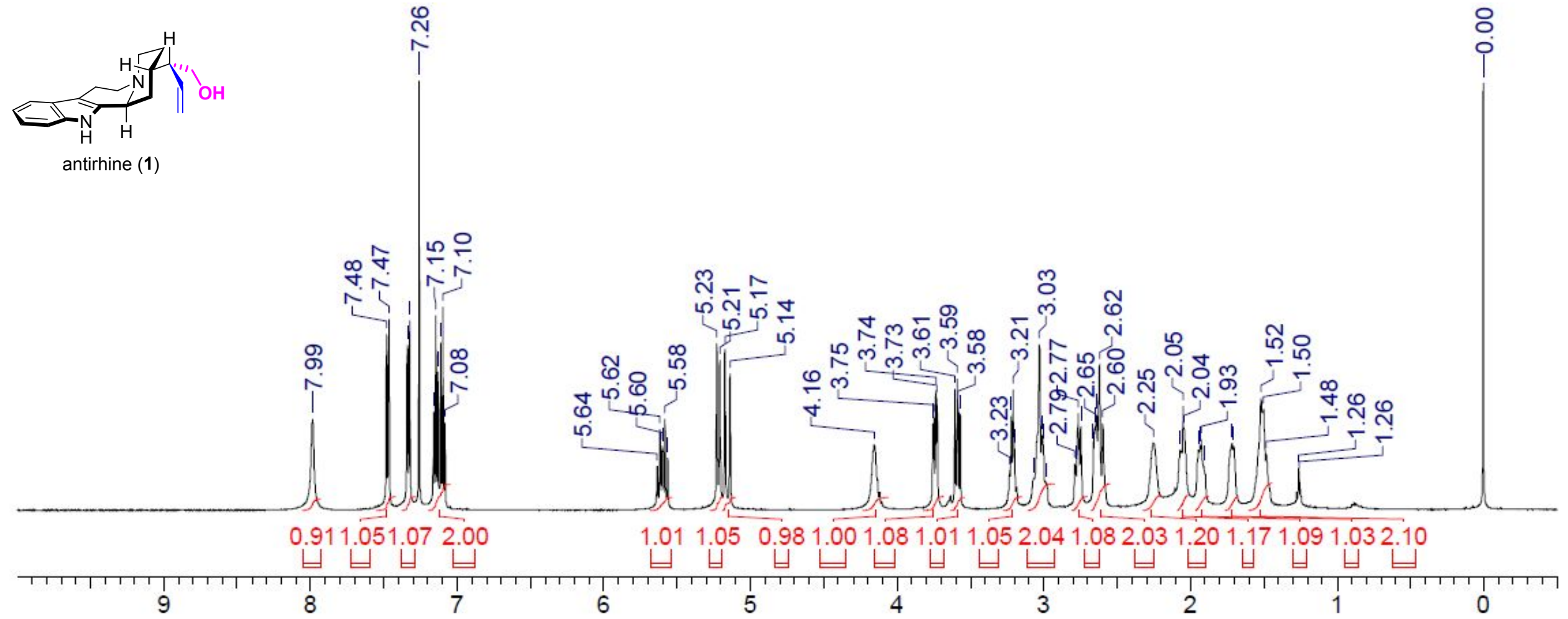


b) ${ }^{13} \mathrm{C}\left\{{ }^{1} \mathrm{H}\right\}$ NMR Spectrum (in $\mathrm{CDCl}_{3}, 125 \mathrm{MHz}$ )

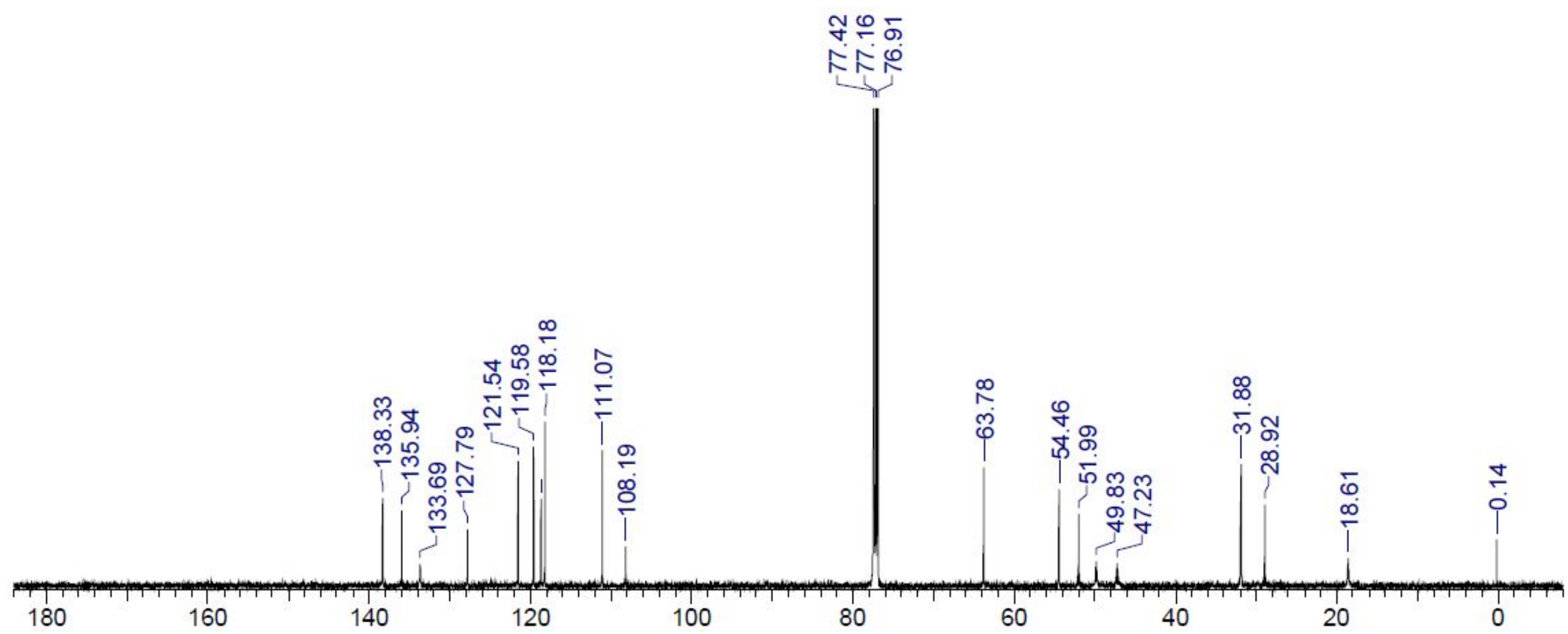

